\title{
Ion multi-nose structures observed by Cluster in the inner Magnetosphere
}

\author{
C. Vallat ${ }^{1,3}$, N. Ganushkina ${ }^{2}$, I. Dandouras ${ }^{3}$, C. P. Escoubet ${ }^{1}$, M. G. G. T. Taylor ${ }^{1}$, H. Laakso ${ }^{1}$, A. Masson ${ }^{1}$, \\ J.-A. Sauvaud ${ }^{3}$, H. Rème ${ }^{3}$, and P. Daly ${ }^{4}$ \\ ${ }^{1}$ ESTEC, European Space Agency, 2201 AZ Noordwijk, The Netherlands \\ ${ }^{2}$ Finnish Meteorological Institute, 11010 Helsinki, Finland \\ ${ }^{3}$ Centre d'Etude Spatiale des Rayonnements, Systeme Solaire, Toulouse, France \\ ${ }^{4}$ Max Planck Institue for Solar System Research, Katlenburg-Lindau, Germany
}

Received: 22 May 2006 - Revised: 24 November 2006 - Accepted: 5 December 2006 - Published: 1 February 2007

\begin{abstract}
During the last 30 years, several magnetospheric missions have recorded the presence of narrow proton structures in the ring current region. These structures have been referred as "nose-like" structures, due to their appearance when represented in energy-time spectrograms, characterized by a flux value increase for a narrow energy range.
\end{abstract}

Cluster's polar orbit, with a $4 R_{E}$ perigee, samples the ring current region. The ion distribution functions obtained in-situ by the CIS experiment (for energies of $\sim 5 \mathrm{eV} / \mathrm{q}$ to $40 \mathrm{keV} / \mathrm{q}$ ) reveal the simultaneous presence of several (up to 3) narrow nose-like structures. A statistical study (over one year and a half of CIS data) reveals that double nose structures are preferentially observed in the post-midnight sector. Also, the characteristic energy of the nose (the one observed at the lower energy range when several noses occur simultaneously) reveals a clear MLT dependence during quiet events $\left(K_{p}<2\right)$ : a sharp transition in the energy range occurs in the pre-noon sector. Moreover, the multi-nose structures (up to 3 simultaneous noses) appear regardless of the magnetospheric activity level and/or the MLT sector crossed by the spacecraft.

Numerical simulations of particles trajectories, using large-scale electric and magnetic field models are also presented. Most of the features have been accurately reproduced (namely the single and double noses), but the triple noses cannot be produced under these conditions and require to consider a more complex electric field model.

Keywords. Magnetospheric physics (Energetic particles, trapped; Magnetospheric configuration and dynamics; Plasma convection)

Correspondence to: C. Vallat

(claire.vallat@esa.int)

\section{Introduction}

During the last 30 years, several inner magnetospheric missions have recorded the presence of various fine structures in the proton spectrograms, which have been referred as "noselike" structures due to the shape of the created features in the energy-time spectrograms. These features are characterized by a deeper inward penetration of particles coming from the tail at a given energy. This global "nose-structure" denomination can however regroup several different formation processes.

Using data issued from the "Explorer 45" (equatorial orbit), Smith and Hoffman (1974) first observed a flux increase of protons in the $15-20 \mathrm{keV}$ energy range, down to $\mathrm{L}=3$ or 4 (i.e. at lower altitude than the plasmapause location), in the evening sector and associated with substorms. This penetration of plasma issued from the plasma sheet was explained as a combination of specific energy that allows particles to penetrate more inward as explained by the adiabatic motion of particles (conservation of the first two invariants) in stationary electric (corotation plus convection) and magnetic fields, together with the existence of forbidden regions for other energy ranges.

Using the same data, Ejiri et al. (1980) described the phenomenon responsible for these structures as a two steps process: first plasma issued from the plasma sheet is injected inward by a relatively weak convective electric field. Then this field increases suddenly, leading to an energy dispersion of the ions. These nose-like structures were thus referred as "substorm nose structures". Ejiri et al. (1980) observed that a minimum AE threshold of $250 \mathrm{nT}$ was necessary to observe substorm nose structures at these distances.

Moreover, some similar features have also been observed on the Polar CAMMICE data during even moderate activity $(\mathrm{AE}=150-250 \mathrm{nT})$. Ganushkina et al. (2001) demonstrated that large-scale electric field changes are not sufficient to explain the fast formation (less than one hour) of these "intense

Published by Copernicus GmbH on behalf of the European Geosciences Union. 
nose events", as named by the authors. They showed that local pulses in the electric field (up to $4 \mathrm{mV} / \mathrm{m}$ ) associated with substorm dipolarization are necessary to explain such rapid formation time and radial location of the observed features.

Some nose-shaped structures were observed by Akebono at altitudes up to $10000 \mathrm{~km}$. Shirai et al. (1997) interpreted them in terms of mono-energetic ion drop off. These gaps, which appear at about $10 \mathrm{keV}$, were observed over a wide latitudinal range at almost constant energy. This gap comes usually together with a simultaneous gap at lower energy, driving to the creation of another type of nose-like structure, usually at lower energy ranges than the substorm nose structures (i.e. below $10 \mathrm{keV}$ ). Moreover, these structures (that will be referred in the following as "stationary nose structures") appear regardless of the magnetospheric activity level. Using an ion drift trajectory model, Shirai et al. (1997) were able to reproduce such structures, and showed that they are the consequence of the open/close character of the particle orbits: at the Akebono location, corotation electric field is sufficient to close the orbit (around the Earth) of low energy particles which are drifting eastward. Also, the magnetic field gradient is strong enough to close the orbits of high energy particles which are drifting westward. As a consequence, for these energies, particles are not continuously supplied from the tail region. However, particles with intermediate energies are on open orbits (neither corotational field nor magnetic field gradient permit to close their orbits around the Earth), creating the nose structures observed on the Akebono data.

Earlier on, Lennartsson et al. (1979), by analyzing ISEE-1 data, demonstrated that in the morning sector the ion velocity distribution was presenting a hole for a given energy range. This hole was explained in terms of the very long time of residence of particles with a given energy, for which the electric field action (leading to an eastward drift of protons) and the magnetic field action (leading to a westward drift of protons) are antagonistic and almost compensate each other. The drastically decreased drift velocity of this population creates a spectral gap in some sectors of the magnetosphere, known also as "stagnation dip".

More recently, Kovrazhkin et al. (1999) listed the different types of gaps observed on the ION spectrograms onboard the INTERBALL spacecraft: the one described by Shirai et al. (1997) on Akebono data and the one described by Lennartsson et al. (1979), corresponding to the very long time of azimuthal drift of particles for a given energy.

Double nose structures (referred in the following as "split nose"), as seen in the morning sector by the ION experiment onboard Interball-2, were studied by Buzulukova et al. (2003). They are interpreted by the authors as the result of a superposition in the spectrograms of a single stationary nose (Shirai et al., 1997) together with the gap described and analyzed by Lennartsson et al. (1979). This gap, which was observed in the morning and pre-morning sector, is created at energies within the nose energy range, leading to a split of the stationary nose into two parts.
Polar also recorded multi bands (more than 2) of ions. This led to several interpretations: on one hand $\mathrm{Li}$ et al. (2000) explained it as the result of ion drift echoes following an increase of the convection electric field. Particles are injected deeply inward, following the electric field increase, and they start to disperse, high energy particles drifting faster around the Earth than low energy ones, their population superposing the slower one locally. On the other hand, Ebihara et al. (2004) interpreted this feature as the result of two combined processes: a change in the convection electric field together with a change in the distribution function of the source to explain the low energy particles features. They showed that a change in the convection electric field can move the nose energy range to higher or lower energies, leading to the simultaneous observation of the "old" nose (created before the convection electric field change) together with a new one.

This variety of structures, presenting roughly the same signatures on the energy-time spectrograms issued from several missions, are thus thought to have different origins and to appear at restricted locations and under specific activity conditions. Their frequency of appearance is however still under question, as well as the dominant mechanisms involved in their formation.

Recent data obtained onboard the Cluster spacecraft by the CODIF/CIS ion mass spectrometers reveal also the presence of different types of nose structures, for any MLT sector or magnetospheric activity level. Here we present a statistical study based on about 163 perigee passes of the Cluster spacecraft, aiming to extract the main characteristics of these features and to determine where they appear preferentially. Then, using numerical modeling, we try to understand the main process(es) responsible for the creation of such structures, and what are the most influent parameters on the structure's shape. After a brief reminder concerning the Cluster mission and its onboard plasma experiments (Sect. 2), we present CIS observations of single and multi-nose structures observed at different MLT locations (Sect. 3). Then, based on more than one year and a half of data, a statistical study over 163 Cluster perigee passes is presented (Sect. 4). Finally, particle trajectory tracing is performed to understand the main mechanisms involved in the structures formation, and the influence of magnetic and large scale electric fields changes on the structures observed (Sect. 5).

\section{Instrumentation}

\subsection{Cluster}

The Cluster mission is composed of four spacecraft launched on closely spaced elliptical polar orbits, with a perigee at about $4 R_{E}$ and apogee at about $19.6 R_{E}$ (Escoubet et al., 2001). This allows Cluster to cross the ring current region from South to North during every perigee pass, and to obtain its latitudinal profile (Vallat et al., 2005). Moreover, due 
to the annual precession of its orbit, Cluster is crossing the equator at all MLT ranges over a year, allowing an equatorial picture of the ring current at the Cluster's perigee distance.

\subsection{CIS}

The Cluster Ion Spectrometry (CIS) experiment on board Cluster, which consists of the two complementary spectrometers HIA and CODIF, provides the three-dimensional ion distributions with one spacecraft spin (4s) time resolution (Rème et al., 2001). The mass-resolving spectrometer CODIF provides the ionic composition of the plasma for the major magnetospheric species $\left(\mathrm{H}^{+}, \mathrm{He}^{+}, \mathrm{He}^{++}\right.$and $\left.\mathrm{O}^{+}\right)$, from the thermal energy to about $\sim 40 \mathrm{keV} / \mathrm{e}$, covering thus a large part of the ring current energy range (Williams et al., 1987). HIA does not offer mass discrimination but has a better angular resolution $\left(\sim 5.6^{\circ}\right)$ that is adequate for ion beam and solar wind measurements.

\subsection{RAPID}

At higher energies, the RAPID spectrometer (Research with Adaptive Particle Imaging Detectors) permits the detection of suprathermal plasma distributions of electrons (from $\sim 20$ to $\sim 400 \mathrm{keV})$ and ions $(\sim 30 \mathrm{keV} /$ nucleon to $\sim 1500 \mathrm{keV})$ (Wilken et al., 2001). As a consequence, Cluster is capable of sampling the entire ring current energy range during its perigee pass (Williams et al., 1987).

\section{Cluster observations}

\subsection{February 2002: single nose}

During the 22 February 2002 perigee pass, Cluster is coming from the Southern lobe through the plasma sheet into the ring current region. The $D_{s t}$ index of $-6 \mathrm{nT}$ reveals a very low ring current activity during the Cluster equatorial crossing, at 00:00 MLT. The AE index remains between 100 nT and $250 \mathrm{nT}$ during the $10 \mathrm{~h}$ prior to the event. Cluster SC4 data are presented in Fig. 1. This figure represents, from top to bottom: the RAPID proton flux (between 27.6 and $1107 \mathrm{keV}$, with a data gap occurring just before the perigee pass), the proton spectrogram in particle flux units as measured by CODIF, the pitch angle distribution of protons for the $\sim 0$ to $10 \mathrm{keV}$ energy range, the $\mathrm{O}^{+}$spectrogram in flux units and the $\mathrm{O}^{+}$pitch angle distribution for the $\sim 0$ to $10 \mathrm{keV}$ energy range. L-shells values, radial distance and invariant latitudes are shown at the bottom of the figure. The red arrow indicates the equatorial crossing.

Arriving from the Southern lobe, Cluster 4 encounters a boundary at 14:28 UT, characterizing its entry into the near Earth plasma sheet. The proton population observed by CODIF has an isotropic distribution function. However, at 14:50 UT, once Cluster arrives at lower latitudes
( $\mid$ Ilat $\mid=68.9^{\circ}$, Southern hemisphere), a field aligned population appears, characterizing ion beams up-flowing from the ionosphere and remaining until 14:58 UT ( $\mid$ Ilat $\mid=67.4^{\circ}$ ).

After 14:50 UT, RAPID is observing the smooth entrance of the spacecraft in the ring current, starting from lower energy particles (around $30 \mathrm{keV}$ ) and extending up at all energy ranges from 14:58 UT. Please note that for this event RAPID data were no longer available when approaching the equator.

After 15:30 UT, an ion gap is present from $19 \mathrm{keV}$ and above in the CODIF spectrogram, which extends to the lower energies with decreasing latitude of the spacecraft. This gap persists down to the equatorial plane both in the Southern and Northern hemispheres. A gap at lower energy ranges (from thermal energies up to $5.5 \mathrm{keV}$ ) appears abruptly at 15:39 UT $\left(\mid\right.$ Ilat $\left.\mid=61.6^{\circ}\right)$. These two simultaneous gaps result in the nose structure in the CODIF spectrogram, whose energy is situated between 5.7 and $7.2 \mathrm{keV}$. The gap is also visible by RAPID, characterized by a decrease in the flux in the 27-68 keV channel as the spacecraft approaches the equatorial plane. After the traversal of the equatorial plane in the Northern hemisphere, the nose structure is still observed.

The main boundaries (i.e. lobe/near Earth plasma sheet and near Earth plasma sheet/ring current) are also observed in the $\mathrm{O}^{+}$spectrograms. Moreover, up-flowing ions issued from the ionosphere are seen in the spectrograms (14:50 to $14: 58 \mathrm{UT}$, |Ilat| between $68.9^{\circ}$ and $67.4^{\circ}$, respectively) and present an inverted "V" structure. Once inside the Plasmasphere region (deduced from the CODIF-RPA data, see Sect. 7 and Fig. 13), a nose structure is also observed for $\mathrm{O}^{+}$, with an energy range between 5.7 and $9.5 \mathrm{keV}$. Unfortunately, the reduced energy resolution of the $\mathrm{O}^{+}$data does not allow us to unambiguously determine if both populations $\left(\mathrm{O}^{+}\right.$and $\left.\mathrm{H}^{+}\right)$ present the nose structure at exactly the same energy. However, both spectrograms $\left(\mathrm{H}^{+}\right.$and $\left.\mathrm{O}^{+}\right)$have a similar appearance.

\subsection{November 2001: "double" nose}

The 21 November 2001 perigee pass occurs in the morning sector (MLT 07:00), and reveals a somehow different feature than the 22 February 2002 one. The $D_{s t}$ index of $-12 \mathrm{nT}$ characterizes the low activity level of the ring current, but the AE index reveals some substorm activity previous to the pass (4 to $14 \mathrm{~h}$ before, not shown). Cluster data are presented in Fig. 2. Approaching from the Southern lobe, Cluster crosses the dayside boundary layer at 19:09 UT (MLT 8.5). This region is characterized by a relatively isotropic distribution of ions (seen from $\sim 19: 30 \mathrm{UT}$ ) with energies of a few $\mathrm{keV}$ to a few tens of $\mathrm{keV}$, and a density $<5 \mathrm{~cm}^{-3}$.

At about 20:30 UT, $\mid$ Ilat $\mid=72^{\circ}$, the spacecraft enter the ring current region, signified by the appearance of trapped $\left(\alpha \sim 90^{\circ}\right)>10 \mathrm{keV}$ ions. We note that the trapped population is not obvious in the pitch angle plot of Fig. 2 due to the broad energy range. By examining the RAPID proton data, we note that the low energy part of the ring current appears 


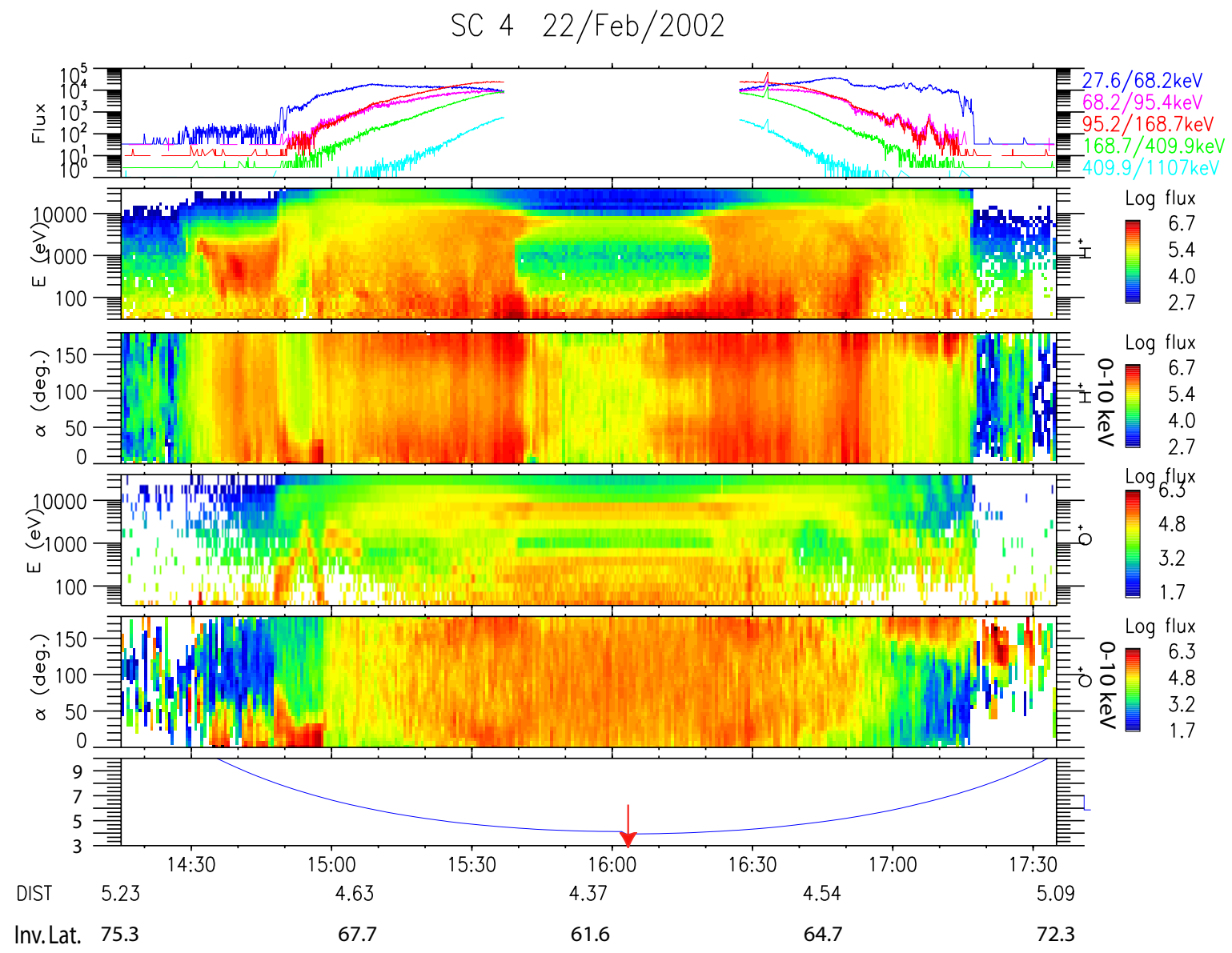

Fig. 1. SC4 particles data for the 22 February 2002 event. From top to bottom: RAPID proton flux in flux units, CIS/CODIF proton flux in flux units, the proton pitch angle distribution in flux units for the $0-10 \mathrm{keV}$ energy range, the $\mathrm{O}^{+}$flux in flux unit, the $\mathrm{O}^{+}$pitch angle distribution in flux unit for the 0 to $10 \mathrm{keV}$ energy range and the L-shell values crossed by the spacecraft. Radial distance in $R_{E}$ and invariant latitudes are indicated at the bottom of the plot. Red arrow indicated the equator crossing.

first, followed by an increase of the fluxes at higher energy ranges as Cluster crosses lower L-shells. Near the Equator, the maximum of the flux is observed within the 95.4 to $168.7 \mathrm{keV}$ energy channel of the RAPID instrument. Since Williams et al. (1987) stated that $90 \%$ of the ring current is carried by particles with energy from 15 to $250 \mathrm{keV}$, this flux maximum is characteristic of this population. It is also worth noting that, along the Cluster path and for the overall ring current energy range (from $10 \mathrm{keV}$ to $500 \mathrm{keV}$ ), lower energy particle fluxes peak at higher L-shells. This has already been observed by AMPTE/CCE/CHEM at all MLT sectors and for energies above $20 \mathrm{keV}$ (Milillo et al., 2001).

At lower energies (i.e. below $10 \mathrm{keV}$ ), several populations seem to emerge: on the one hand, down to $|\mathrm{Ilat}|=63^{\circ}$ (21:17 UT, Southern Hemisphere) and $|\mathrm{Ilat}|=60.5^{\circ}$ (22:17 UT, Northern Hemisphere), very narrow structures are seen at low energies (up to $1 \mathrm{keV}$ ), presenting a butterfly pitch angle distribution (not shown).
On the other hand, a trapped population co-exists at medium energies (between 1.5 and $7 \mathrm{keV}$ ), which forms a nose-like structure at low L-shells, centered around $3.8 \mathrm{keV}$ and stationary between the inbound and the outbound of the Cluster orbit. Above this energy, another stationary nose-like structure appears around $16 \mathrm{keV}$, extending down to $\mid$ Ilat $\mid=62.6^{\circ}(21: 18 \mathrm{UT})$. These two noses co-exist locally. However, the equatorial edge of the lower energy nose is situated at lower latitudes.

The gap separating the two noses persists throughout the perigee pass, from $|\mathrm{Ilat}|=69.9^{\circ}$ in the Southern Hemisphere (20:40 UT) to $|\mathrm{Ilat}|=70.4^{\circ}$ in the Northern Hemisphere (23:13 UT), its characteristic energy increasing with decreasing latitude.

\subsection{April 2002: "triple" nose}

Multi noses structures are also evident in the CODIF spectrograms during several Cluster perigee passes. Data for the 11 April 2002 perigee pass (MLT 21:00) are shown in Fig. 3. 


\section{SC 4 21/Nov/2001}

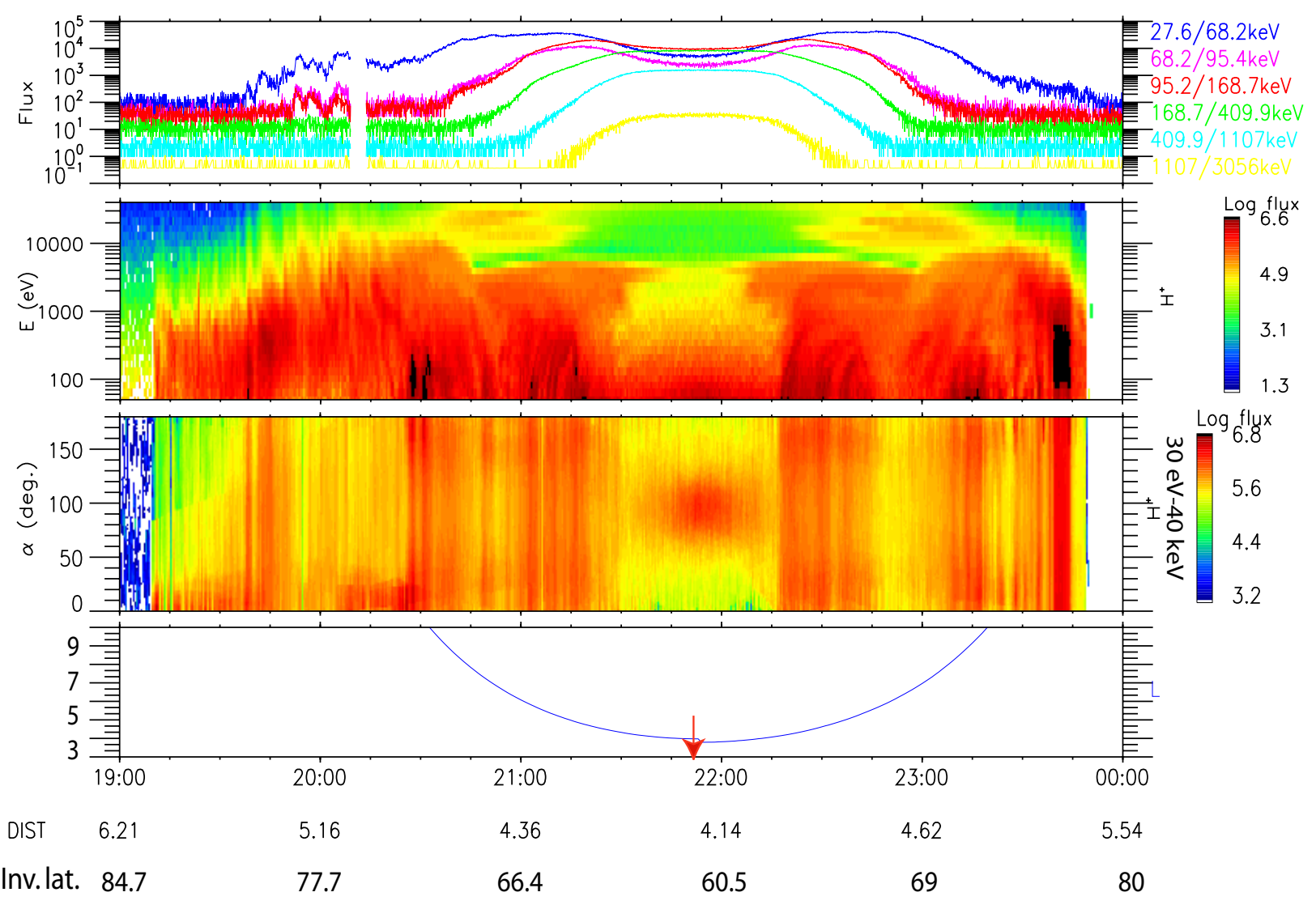

Fig. 2. SC4 particles data for the 21 November 2001 event. From top to bottom: RAPID proton flux in flux units, CIS/CODIF proton flux in flux units, the proton pitch angle distribution in flux units for the $30 \mathrm{eV}-40 \mathrm{keV}$ energy range and the L-shell values crossed by the spacecraft. Radial distance in $R_{E}$ and invariant latitudes are indicated at the bottom of the plot. Red arrow indicated the equator crossing.

This event also takes place during a very quiet period, with a positive $D_{s t}$ index during more than two days preceding the equatorial crossing by the spacecraft $\left(D_{s t}=21 \mathrm{nT}\right.$ during the crossing). The $K_{p}$ index, always below 2 during the $12 \mathrm{~h}$ prior to this event, confirms these quiet magnetosphere conditions.

Initially in the magnetosheath, Cluster crosses the boundary layer before entering the ring current region at about 04:14 UT (|Ilat $\mid=67.2^{\circ}$, Southern Hemisphere).

At energies higher than $3 \mathrm{keV}$, several nose structures are observed simultaneously: at least two during the inbound part of the orbit and three during the outbound (shown by the black arrows).

The poleward edges of the two inbound noses start at $\mid$ Ilat $\mid=66.4^{\circ} \quad$ (Southern hemisphere, 04:21 UT), with the lower energy nose penetrating deeper $\left(\mathrm{L}_{\min }=4.63\right.$ for the nose centered around $5 \mathrm{keV}$ and $\mathrm{L}_{\min }=4.9$ for the one centered around $18 \mathrm{keV}$ ). During the outbound part of the orbit, a third nose structure is detected at even higher energy (about $26 \mathrm{keV}$ ), albeit with a dispersion looking smaller than the other noses (however at those latitudes, this corresponds to a dispersion between $\mathrm{L}_{\min }=5.64$ and $\mathrm{L}_{\max }=6.22$ ).

In spite of a higher level of background in the $\mathrm{O}^{+}$spectrograms in the radiation belts (due to the longer time of flight of the $\mathrm{O}^{+}$in the detection system), the first nose structure (characteristic energy below $10 \mathrm{keV}$ ) is also seen in the oxygen data. A second nose is present at higher energy during the inbound (between 15 and $20 \mathrm{keV}$ ) but no other nose is detected for this population. However, since the energy discrimination is not as good for oxygen as for protons (16 energy steps for $\mathrm{O}^{+}, 31$ for $\mathrm{H}^{+}$), it is possible that such narrow energy structures are not resolved in the $\mathrm{O}^{+}$.

These three events, in spite of the similar activity of the magnetosphere and the same radial location of the spacecraft, are presenting strongly different features, i.e. single, double or triple nose structures. 


\section{SC 4 11/Apr/2002}
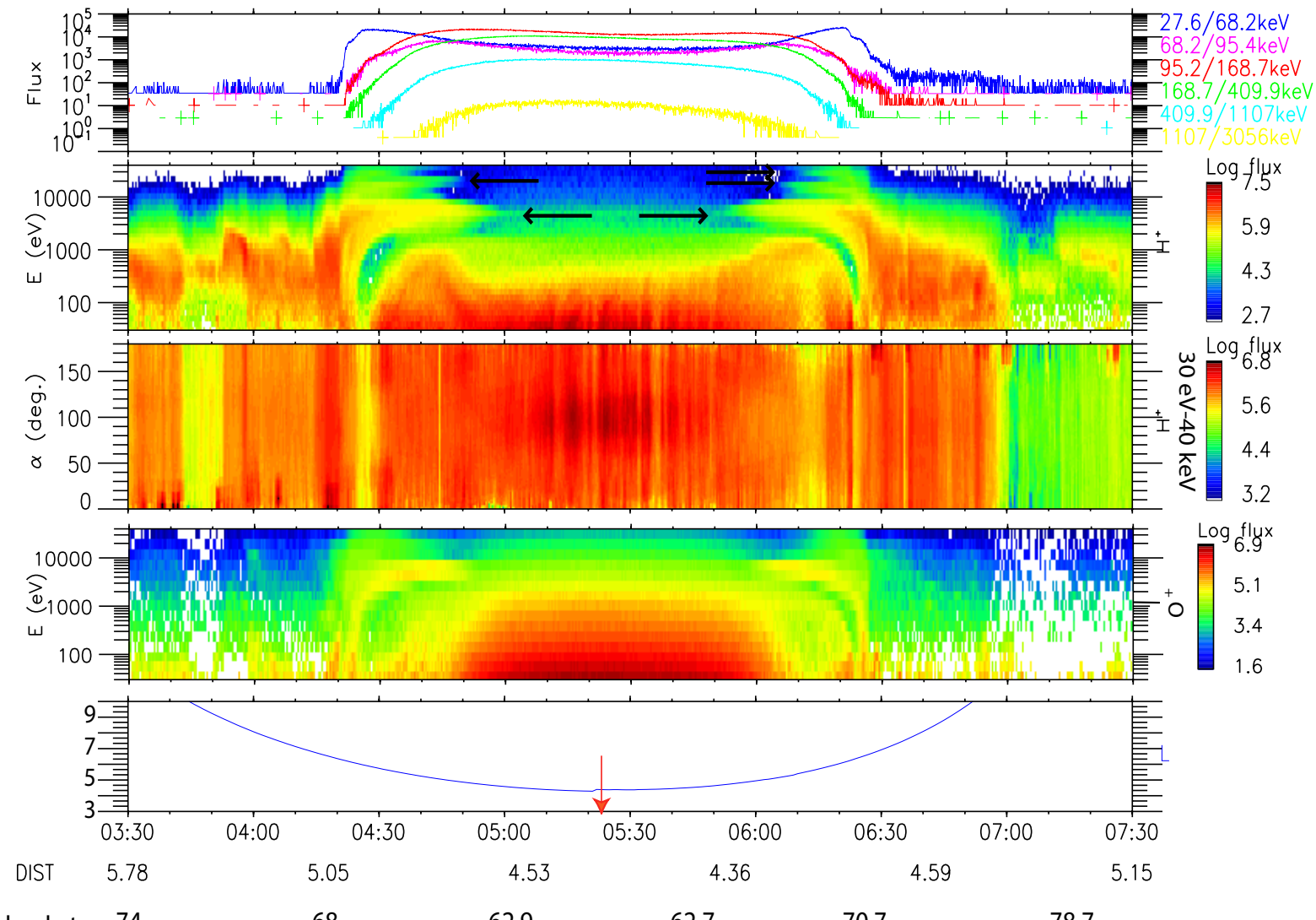

Inv. Lat. 74

68

62.9

62.7

70.7

78.7

Fig. 3. SC4 particles data for the 11 April 2002 event. From top to bottom: RAPID proton flux in flux units, CIS/CODIF proton flux in flux units, the proton pitch angle distribution in flux units for the $30 \mathrm{eV}-40 \mathrm{keV}$ energy range, the $\mathrm{O}^{+}$flux in in flux units and the L-shell values crossed by the spacecraft. Radial distance in $R_{E}$ and invariant latitudes are indicated at the bottom of the plot. Red arrow indicated the equator crossing.

\section{Statistical study}

To investigate the distribution of the different types of structures, a database has been created based on 163 perigee passes of Cluster SC 4 (or SC1 when data from SC4 were not available), between the 5 October 2001 and the 8 May 2003. Data acquired after May 2003 were not taken into account in this study: due to radiation belts background contamination (as a consequence of the instrument degradation), CODIF does not allow accurate enough observations of the narrow energy structures near perigee..

The perigee distance did not change significantly during this time interval, evolving from $\mathrm{R}_{p}=4.13 R_{E}$ to $\mathrm{R}_{p}=4.04 R_{E}$ between the first and the last event, respectively. Within this time interval, the perigee of SC4 was always situated between 4 and $4.5 R_{E}$.

Since an automatic recognition of the nose structures is not feasible due to the "zoo" of structures co-existing at the Cluster perigee location, we analyzed every perigee pass vi- sually and recorded every nose observed on the proton spectrograms. We made a distinction between events presenting a single nose structure, double nose structure and 3 noses structure (maximum number of noses recorded by CODIF during a single pass). Approximately 60 perigee crossings during this period were not considered for the study: they included events presenting an uncertainty concerning the number of bands observed (only a few events), and events for which the radiation belts background was too high to determine unambiguously if a structure was present or not.

When inbound and outbound parts of a single orbit didn't reveal the same number of noses (only for a very few cases, such as the 11 April 2002 event), we chose to consider only the part of the orbit having the higher number of noses. Moreover, events for which the higher energy boundary of a structure was not visible on the CIS spectrograms (its energy range extending above the upper energy limit of the instrument) were not taken into account. Finally, wedge-like structures (Ebihara et al., 2001) were not considered since 
their characteristic energy, together with the structure they are forming on the spectrograms, is somehow different from the nose ones (see Yamauchi et al., 2006).

For each of the 163 events the number of noses, their characteristic energy and the MLT sector crossed by the spacecraft has been noted. Moreover, the $K_{p}$ and $D_{s t}$ indices associated to each event have also been listed. Over the 163 perigee passes listed, 49 were not revealing any nose structure, 71 were presenting a single nose structure, 40 were presenting a double nose structure and only 3 events revealed three simultaneous noses.

4.1 Normalized occurrence of the numbers of simultaneous structures $(0,1,2$ and 3$)$ for various $\left(K_{p}\right.$, MLT) couples

Figure 4 presents the normalized occurrence of the number of energy structures per $K_{p}$ index range. A distinction has been made between events presenting no nose structure (yellow), one nose (cyan), two noses (purple) or three noses structures (red).

Without further analysis, it already appears that for high activity levels, the lack of nose seem to be the most frequent case. This can be understood since during high activity periods (several successive substorms for example) freshly injected particles can hide the previously formed stationary nose structures.

During low and moderate activity periods, single nose structures appear preferentially, but double nose is also often observed.

Figure 5 presents the normalized occurrence of the number of energy structures per $K_{p}$ index range, separately for each of the four quadrants of the Magnetosphere (from MLT $=00: 00$ to 06:00, from MLT $=06: 00$ to $12: 00$, from MLT $=12: 00$ to $18: 00$ and from MLT=18:00 to 00:00). The normalization is made over the number of passes per quadrant and per $K_{p}$ range.

These plots reveal the overall changing distribution of the number of noses observed in the CODIF spectrograms with respect to the MLT sector crossed by the spacecraft. During low activity periods, double noses structures seem to occur frequently, especially in the post-midnight and morning sectors.

Events for which CODIF recorded 3 noses remain exceptional and no statistics can be made concerning this type of feature.

Concerning high activity events $\left(K_{p}>4\right)$, zero and single nose structures are predominant. Nevertheless, the poor statistics available for this kind of event is especially bad in the 06:00-12:00 and 12:00-18:00 MLT sectors (dayside). As a consequence, no conclusion can be made per MLT sector.

\subsection{Characteristic energy of the nose vs. MLT}

Figure 6 shows the mean energy of the nose structure (at its equatorial edge) for each of the 163 perigee passes, as
All MLT: Normalized occurence of structure per Kp

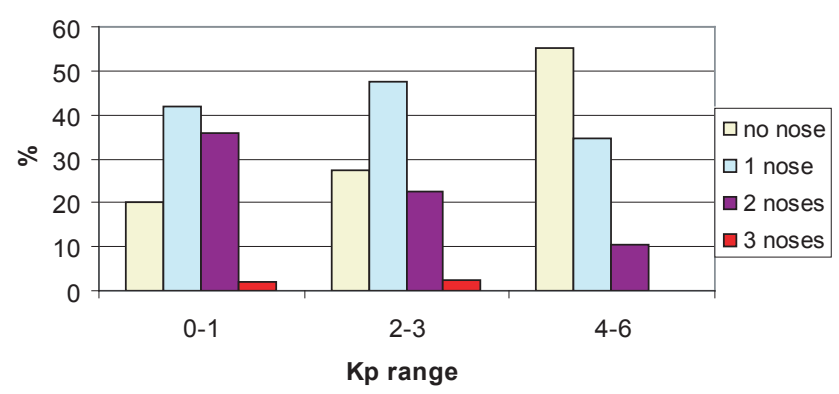

Fig. 4. Normalized occurrence of the number of structures per $K_{p}$ values.

a function of the MLT sector crossed by Cluster. When several nose structures occur simultaneously, the associated energies are plotted, i.e. colored marks are used to distinguish the lowest energy range nose (blue marks), the second one (pink squares) and the third one (yellow triangles) observed by CODIF. Some inaccuracies on the energy determination can occur since the energy resolution of the instrument is averaged over the mean energy of each energy bin.

Some remarks emerge:

This is the first time that multi-nose structures are observed over such a wide MLT range. Moreover, three simultaneous noses are also observed occasionally in the evening sector (the few events presenting such structures are situated in the 21:00-04:00 MLT sector).

In this plot (all activity levels included), we do not see any clear relationship between the nose energy and the MLT sector where they are observed. However, if we make a distinction between quiet events (i.e. with $K_{p}$ index up to 1 ) and disturbed ones, we get the results shown in Figs. 7 and 8.

Even if the statistics are much more reduced, this quiet event selection (Fig. 7) presents a clearer trend of the energyMLT dependence:

Focusing on the energy range of the first nose-like structure (blue marks), we clearly observe an MLT dependence of this characteristic energy range with the nose energy decreasing from $\sim 15 \mathrm{keV}$ down to $\sim 1 \mathrm{keV}$, counterclockwise from the pre noon sector (around MLT 10:00).

Using a linear fit for the lower energy noses (see Fig. 7), we can deduce a relation between the energy of the nose and the MLT sector:

$E(\mathrm{keV})=-0.3276 \times \mathrm{MLT}_{10}+14.975$

and MLT $_{10}=$ MLT in hours in the dusk half sector (from MLT $=10$ to MLT $=24$ ), and MLT $_{10}=$ MLT +24 otherwise. The correlation coefficient is, in that case, $r^{2}=0.6339$. A similar trend is also seen in the pink marks, representing the energy range of the higher energy nose (when observed). 
MLT= 12-18: Normalized occurence of structures per Kp

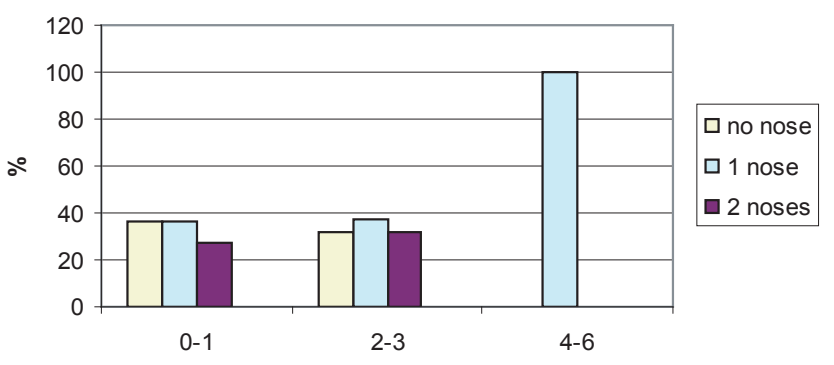

(a)

\section{MLT=6-12: Normalized occurence of structures} per Kp

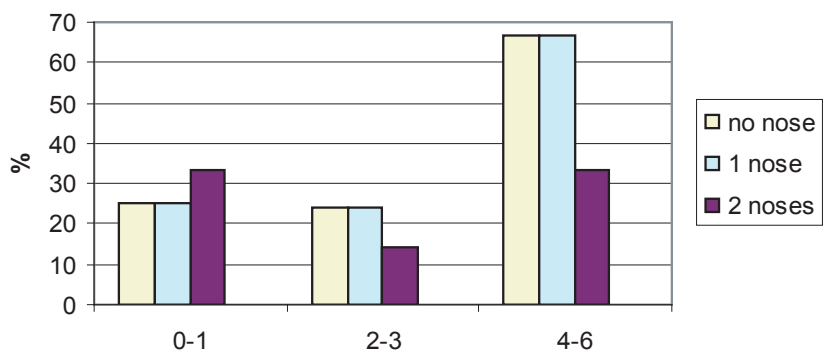

(b)

Kp range
MLT=18-24: Normalized occurence of structures per Kp

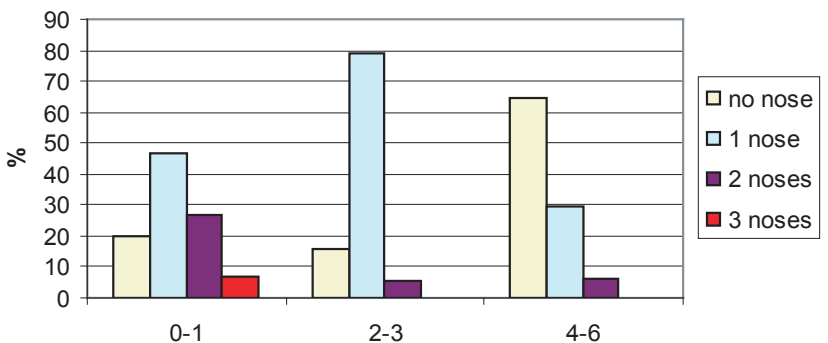

(c)

Kp range

$M L T=0$ to 6 :

Normalized occurence of structures per Kp

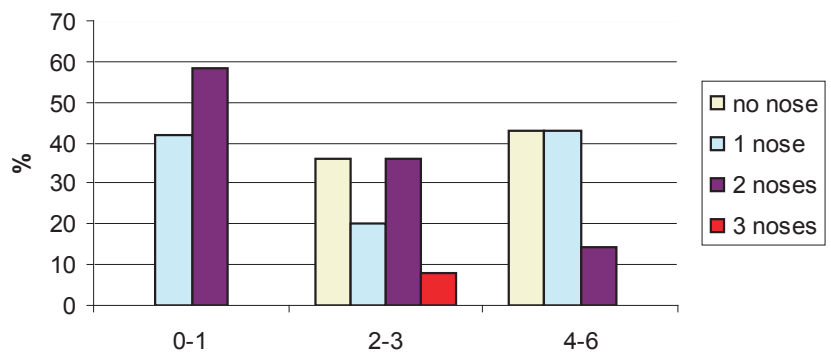

(d)

Kp range

Fig. 5. Normalized occurrence of structure type per (MLT, $K_{p}$ ) couples:

(a) Upper left: MLT=12:00 to 18:00

(b) Lower left: MLT=18:00 to 00:00

(c) Upper right: MLT $=06: 00$ to 12:00

(d) Lower right: MLT=00:00 to 06:00

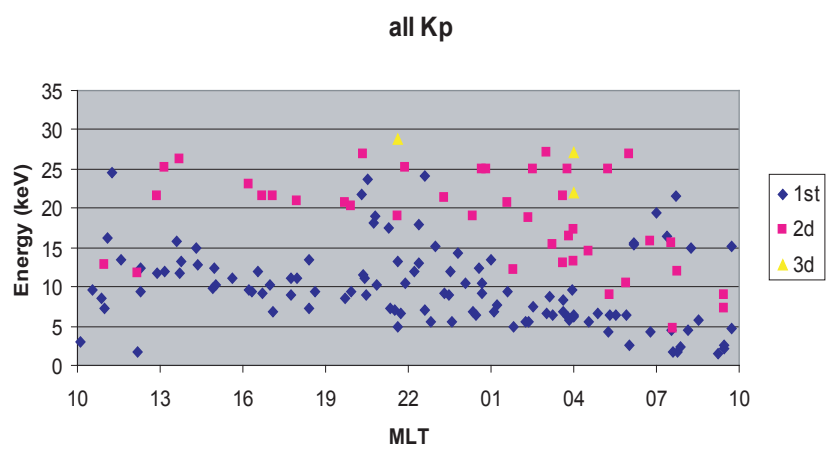

Fig. 6. Characteristic energy of each nose-like structure observed by CODIF, as a function of the MLT sector crossed by Cluster. Blue marks represent, when occurring, the nose observed at the lower energy range, whereas pink marks (resp. yellow) represent characteristic energies of the second (resp. third) nose-like structure.

Another way to show the data is to plot $\log \left(\mathrm{E}_{\mathrm{NOSE}}\right)$ with respect to $\mathrm{MLT}_{10}$, with $\mathrm{E}_{\mathrm{NOSE}}$ in $\mathrm{eV}$ :

$\log \left(E_{\text {NOSE }}\right)=-0.0228 \times \operatorname{MLT}_{10}+4.3552$

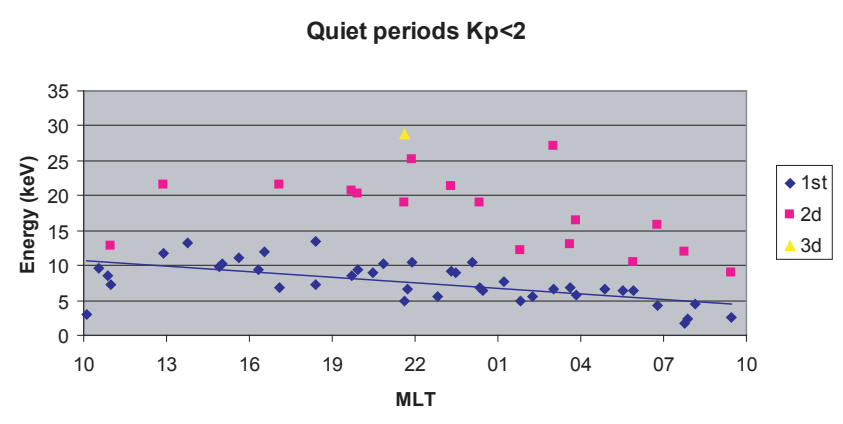

Fig. 7. Characteristic energy of each nose structure vs. MLT, for $K_{p}=0$ and 1 .

with $r^{2}=0.5678$ (plot not shown). However, during more active events (i.e. $K_{p}=2$ or 3 , see Fig. 8 ), we do not observe a trend for the lower energy nose as clear as for more quiet events (see Fig. 7). Even if the same global trend seems to be present, secondary trends are observed locally, especially in the evening (21:00-00:00) and morning (06:00-12:00) sectors (see Fig. 8). 


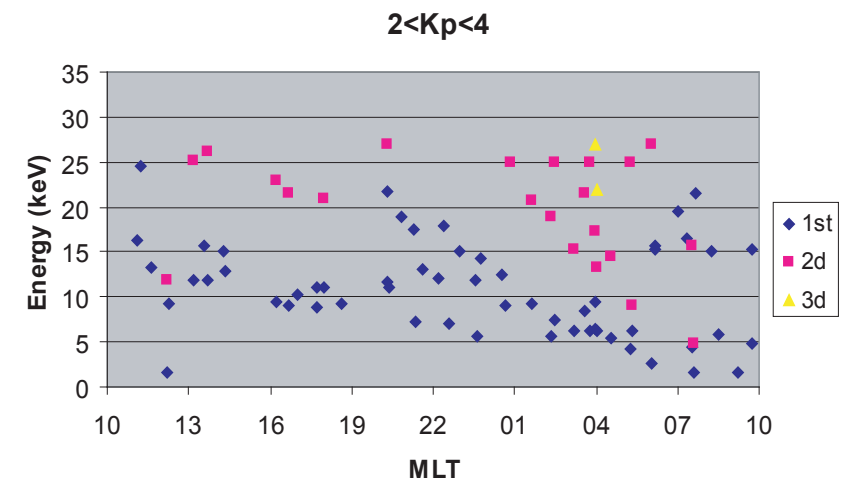

Fig. 8. Characteristic energy of each nose structure vs. MLT, for $K_{p}=2$ and 3 .

In the morning sector, two trends appear for the lowest energy nose: characteristic energy of the nose is found below $5 \mathrm{keV}$ for some events, whereas high energy is also found in that sector, above $15 \mathrm{keV}$. This energy transition occurs roughly in the same MLT sector that the one observed during quiet events (Fig. 7), but this transition seems to occur over a wider MLT range, i.e. from MLT 05:00 to MLT 09:00.

We also note the lack of double nose structures in the statistics for the 21:00 to 01:00 MLT sector. However, characteristic energies vary a lot from one event to the other, reaching values from $5 \mathrm{keV}$ up to $22 \mathrm{keV}$.

4.3 Characteristic energies of the lowest energy nose (for each perigee pass), as a function of MLT and $K_{p}$

Figure 9 shows the energy range of the nose as a function of MLT and $K_{p}$. For events presenting a multi-nose structure, only the lowest energy nose has been considered.

Figure 9 reveals that, even if no linear relationship exists between the nose energy and the $K_{p}$ index, high energy nose structures (i.e. above $15 \mathrm{keV}$ ) are only observed during disturbed periods $\left(K_{p}>2\right)$. The variability of the nose energy is particularly clear in the evening (MLT=20:00 to 00:00) and morning (MLT=06:00 to 11:00) sectors.

\section{Discussion about Cluster data}

\subsection{February 2002}

The 22 February 2002 event presents a clear single nose structure in the proton spectrogram at the Cluster location. Considering that the plasma source is situated in the magnetotail, nose structures, if due to energy dispersion, are expected to be more difficult to observe in the midnight sector, where the time dispersion is shorter than for other MLT sectors (Ejiri et al., 1980). Considering the relatively quiet magnetospheric activity level for this event, as well as the stationarity of the structure between the inbound and the out-

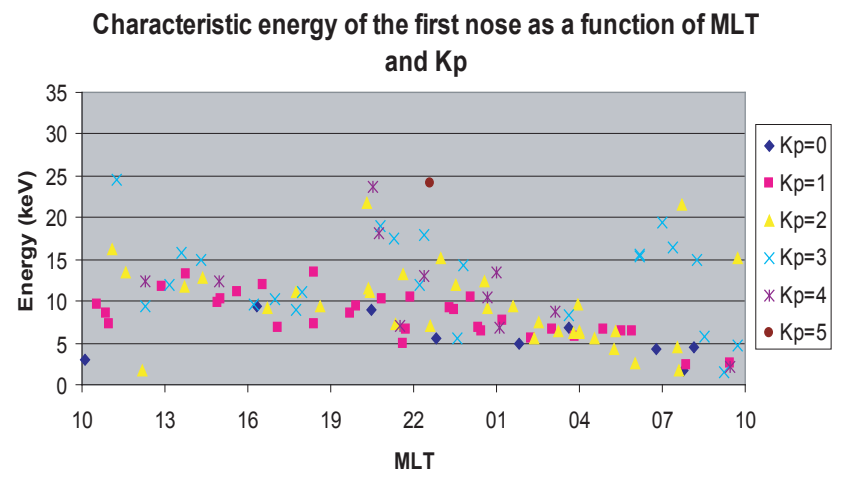

Fig. 9. Characteristic energy of the low energy nose structure vs. MLT, for different $K_{p}$ values.

bound leg of the Cluster orbit and the typical energy range of the observed nose structure (i.e. $6.45 \mathrm{keV}$ in average), this feature seems to be related to the stationary nose structures described in previous papers (Shirai et al., 1997).

The nose structure is also seen in the $\mathrm{O}^{+}$spectrogram. In addition, an inverted "V" structure is observed at higher latitude and at low energies (up to $3 \mathrm{keV}$, Fig. 1). This type of feature has been reported previously (Maggiolo et al., 2006) and was interpreted as the result of local acceleration of the plasma issued from the polar cap.

\section{$5.2 \quad 21$ November 2001}

The 21 November 2001 event presents a more complex structure, characterized by some ion dispersed structures at low energy together with two simultaneous noses like structures at higher energies. The type of dispersion recorded at low energy has already been observed previously, and has been referred as a "wedge-like" dispersion type (Ebihara et al., 2001; Yamauchi et al., 2006). Ebihara et al. (2001) have interpreted these sub-keV structures as the result of temporal and spatial variations in the source region of ions.

At higher energy, the two noses are separated by a gap which persists over a wide latitudinal range, its energy increasing with decreasing latitude. To explain this gap appearance, let's consider the ion drift velocity. This latter can be expressed as the sum of four terms:

$$
\begin{aligned}
\boldsymbol{v}_{d}= & (((\boldsymbol{R} \times \boldsymbol{\Omega}) \times \boldsymbol{B}) \times \boldsymbol{B}) / B^{2}+\boldsymbol{E} \times \boldsymbol{B} / B^{2} \\
& +\left(W_{\perp} \boldsymbol{B} \times \nabla \boldsymbol{B}\right) / q B^{3}+\left(2 W_{/ /} \boldsymbol{r}_{c} \times \boldsymbol{B}\right) / q R_{c} B^{2}
\end{aligned}
$$

where $W_{\perp}=\frac{1}{2} m v^{2}$ is the particle perpendicular kinetic energy, $\boldsymbol{R}$ the position of the particle, $\Omega$ the angular velocity of the Earth's rotation, $R_{c}$ the radius of curvature and $\boldsymbol{r}_{c}$ a unit vector outward from the center of curvature.

The two first terms represent the influence of the corotational and the convection electric field respectively, whereas the last two terms are characterizing the influence of the magnetic field gradient and curvature respectively. The 
terms due to the electric field are independent of the particle charge and energy and lead to an eastward drift for both the electron and the ion populations. The terms due to the magnetic field configuration are charge dependent and cause an eastward drift for electrons and a westward drift for ions. Moreover, since these two terms are energy dependent, it can easily be understood that for a limited energy range of ions, the two sets of terms (i.e. electric field and magnetic field influence) are in conflict and almost compensate each other, causing a strong decrease of the drift velocity for this particular energy range. This will lead to the creation of a gap (or "stagnation dip") at this energy.

At altitudes below $3 R_{E}$, these gaps appeared between 2 to $4 \mathrm{keV}$ in the Interball-2/ION spectrograms, in the morning and dayside sector (Buzulukova et al., 2003). Kovrazhkin et al. (1999) have studied the energy range of this gap as a function of the MLT sector. They demonstrated that the energy of the gap was increasing with decreasing magnetic local time. In the morning sector they have shown that $E_{\text {gap }}$ was appearing between $\sim 5$ and $8 \mathrm{keV}$ for $|\mathrm{Ilat}| \sim 65^{\circ}$, consistently with the one observed by CODIF during this event.

Using a drift loss model, Jordanova et al. (1999) calculated the proton spectra along the Polar orbit and demonstrated that the ion gap observed by Polar was also the consequence of the small azimuthal velocity of particles for a given energy range. Their simulations revealed that at low latitude and at $\mathrm{L}=4.5$, the energy of the gap was centered at $\mathrm{E} \sim 6 \mathrm{keV}$ in the post-noon sector, i.e. slightly above the energy range of the one recorded by CODIF for the 21 November 2001 event (morning sector). This gap energy range evolution with respect to the magnetic local time is consistent with the results of Kovrazhkin et al. (1999).

As a consequence, a possible reasons for the appearance of this double nose on the CODIF spectrogram could be the superposition of a single nose structure together with the gap due to the large time of drift (with energy within the nose energy range). This would lead to a split of the single nose into two noses.

\subsection{April 2002}

The 11 April 2002 event presents 2 noses during the inbound and 3 noses during the outbound part of the orbit. This structure asymmetry is probably a direct consequence of the orbit asymmetry with respect to the tilted magnetic dipole rather than the consequence of a substorm injection, since no activity is recorded during this time interval. Because of this asymmetry, we cannot be certain whether the third nose structure exists on the inbound section of the orbit, due to the upper energy limit of the CODIF instrument and the broad resolution of the lowest RAPID channel, which is not adequate to detect such narrow structures.

\subsection{Statistics}

The statistics made over more than 160 perigee passes of Cluster has revealed a clear dependence of the activity level with the number of noses seen in the CODIF spectrograms, single nose being the most frequent feature during low activity periods, whereas no nose is preferentially observed with increasing activity levels.

Ganushkina et al. (2000) have done a statistical study based on 396 traversals of the inner magnetosphere by Polar. Using the MICS experiment onboard Polar, they listed, as a function of the geomagnetic activity (based on the $K_{p}$ index), the number of intense nose events recorded by the instrument. It appeared that nose structures are more frequent when $K_{p}=3$, the normalized occurrence decreasing linearly with decreasing $K_{p}$. Higher $K_{p}$ values being less frequent, the trend becomes less clear after $K_{p}=3$. The disagreement between our results is mainly explained by the energy threshold differences between both CODIF and MICS instruments: while CODIF records particles with energy as low as a few $\mathrm{eV}$, MICS lower threshold is about $10 \mathrm{keV}$. As a consequence, most of the noses structures observed by CODIF for $K_{p}=0$ and 1 (see Fig. 6) cannot be detected by MICS. On the contrary, since the upper energy threshold of MICS reaches $200 \mathrm{keV}$, a large portion of the intense nose structures are not seen by CODIF, whose energy detection goes only up to $40 \mathrm{keV}$. Moreover, since the nose appears generally at higher energy with increasing activity level (see Fig. 9), the difference between Cluster and the Polar data can be explained.

Finally, Ganushkina et al. (2000) have added a selection criterion based on the flux values to determine intense nose structures. They only considered as nose the structures presenting flux values above $10^{6}\left(\mathrm{~cm}^{2} \mathrm{~s} \mathrm{srkeV}\right)^{-1}$, whereas CODIF reveals nose structures with smaller fluxes. As a consequence, and if we assume that the absolute flux values increase with increasing activity, it is not surprising that the number of intense events increases with increasing $K_{p}$, and that the flux threshold defined by Ganushkina et al. (2001) is more often reached during more active periods.

Our Fig. 5 reveals the most frequent occurrence of double nose structures in the post-midnight and morning sector, during low activity periods. However, if we assume that double noses are often the consequence of a single nose split (i.e. similar to the interpretations by Buzulukova et al., 2003), this kind of distribution can be easily understood. Nevertheless, the split nose structure can only take place under some specific conditions, i.e. when the gap resulting from the enhanced time of residence has enough time to form and where it can be formed. According to Buzulukova et al. (2003), this corresponds to the pre-morning and dayside sector of the magnetosphere. However, theses structures are also seen in the evening sector by CODIF, where they cannot be explained by the split nose theory, since the spacecraft location is too close to the source region to allow the creation of such gaps in that sector. Several interpretations could explain the 
presence of double nose in that sector. First, variations in the azimuthal location of the source: the drift path of particles from the source to the evening sector would be longer, leading to the creation of a gap, and hence split nose in that sector. They could also be due to a change of the global electric field, affecting the nose energy, and leading to the appearance of a nose at different energy, coexisting locally with the one previous to the change (Ebihara et al., 2004). Finally, a modification of the ionospheric conductivity could also lead to a new distribution of the current closure, thus affecting the resulting electric field (Fok et al., 2001). This can lead to twisted paths of particles (Fok et al., 2003), and thus to the appearance of split nose structures in that sector.

It is also worth noting that single nose structures can appear in the morning sector without being necessarily accompanied by this type of gap, since this latter has a formation time of $\sim 15 \mathrm{~h}$ (Kovrazhkin et al., 1999). Thus, the creation of such a gap should require quiet conditions for at least $15 \mathrm{~h}$.

Figures 6,7 and 8 point out the clear dependence of the nose energy range with respect to the MLT sector crossed by Cluster during low activity periods (Fig. 7): there is a general decrease of the nose energy with respect to MLT, starting from about MLT=10:00, counterclockwise. If we assume that all double nose structures are the consequence of a split nose effect, the energy recorded by CODIF for the lowest nose will not necessarily represent the mean energy of the stationary nose. As a consequence, and since we note a lack of double nose structures in the afternoon sector (i.e. right after the transition), a possible explanation for this pre-noon discontinuity could be the appearance in that sector of the gap due to the long time of drift (Buzulukova et al., 2003). This could artificially create a lower energy nose in the morning sector.

Particle trajectories simulations have been performed by Kovrazkhin et al. (1999) for different latitudes. They have shown that for a given latitude, the energy of the gap is decreasing with increasing MLT (from MLT=04:00 to MLT=20:00) as a result of the longer eastward transport of low energy particles. They also showed that with decreasing latitude, the energy of the gap was increasing for a given MLT and the gap was present over a wider range of MLT, as the result of the comparatively larger eastward oriented $\boldsymbol{E} \times \boldsymbol{B}$ drift. The CODIF data do not agree with this study (at least for quiet events), since the gap energy recorded by CODIF is regularly decreasing from the pre-noon rather than from the post-midnight sector. The afternoon and evening sectors are anyway consistent with the simulations presented by Kovrazhkin et al. (1999), and the overall higher energies of the gaps observed by CODIF for a given MLT could be the consequence of the lower latitude of observations. However, simulations made by Kovrazhkin et al. fit pretty well with the more disturbed events observations made by CODIF, where the nose energy starts to decrease from MLT 04:00, as calculated by the authors. One explanation to this discrepancy between CIS observations and Kovrazhkin et al. (1999) model for quiet events could be the azimuthal location of the source. If the source location is azimuthally shifted, the MLT location of the gap for a given energy should also be shifted (in the simplest case of stationary magnetosphere conditions). If the source is situated in the morning sector rather than near midnight, the gap created by the longer time drift should appear at different MLT.

Other studies have also successfully demonstrated the presence of particle source in the morning sector. Studying the wedge-like dispersion structures by CODIF, Yamauchi et al. (2006) demonstrated that the wedge-like structures presenting a large energy range (up to $10 \mathrm{keV}$ ) are formed in the morning sector (07:00-08:00 MLT).

Milillo et al. (2001), using AMPTE/CCE/CHEM data, developed an empirical model describing the average perpendicular proton population fluxes in the equatorial inner magnetosphere. This flux has been described by a multi parametric function. One parameter, namely $\mathrm{COAG} 2_{B}$, describes the basic ion flux at intermediate energy (from 5 to $60 \mathrm{keV}$ ) during quiet magnetospheric activity $(\mathrm{AE}<100 \mathrm{nT}) . \mathrm{COAG} 2_{B}$ is energy and MLT dependent. Its dependence with respect to MLT follows (see Fig. 9 of the Milillo et al. (2001) paper):

$\mathrm{COAG} 2_{B}=-(0.0318 \pm 0.0008) \times h+(3.66 \pm 0.02)$

With $h=$ MLT $-24 \mathrm{n}, \mathrm{n}=0,1$.

This relation is very similar to the relation (1b) we present in Sect. 4.2, $\operatorname{COAG} 2_{B}$ following the same trend as the $\log \left(\mathrm{E}_{\text {NOSE }}\right)$ recorded by CODIF. Both relations reveal the existence of a discontinuity of the nose energy in the dayside sector. This discontinuity at noon was explained by Milillo et al. (2003) as the consequence of a gap created in that sector, within the energy range of the considered population (5-60 keV). However, using AMPTE/CCE data, Milillo et al. (2001) positioned the discontinuity around MLT=12:00, whereas Cluster observed it around MLT=10:00. A possible explanation of this azimuthal shift between the two datasets could be the different Sun activity level during these two periods. Whereas AMPTE/CCE data were recorded close to solar minimum, the Cluster data were recorded close to Solar maximum. As a consequence, the modification of the solar activity could lead to a global change of the convection electric field in the inner Magnetosphere and therefore affect the azimuthal position of the structure formed by the ions.

Our Fig. 9 shows that high energy noses are usually observed only during disturbed periods. However, there is a lack of clear correlation between $K_{p}$ index and nose energy. To explain this, we should notice that all events (i.e. events presenting single, double and triple noses) have been considered here. As a consequence, no distinction has been made between single and split nose structures. Moreover, changing the location of the particle source can affect the nose energy: if the source is moving, the nose energy can change from one event to the other without necessarily having a different $K_{p}$ value. 
Following these results, some questions emerge:

- How can we explain the different azimuthal position of the sharp nose energy range transition (from the noon sector to the morning sector) with increasing activity?

- What process is responsible for the appearance of double and triple noses, especially in the evening sector and during quiet times?

- How is the shape and energy of the nose affected by changes in the electric field, the magnetic field and the particle source distribution?

\section{Simulations}

To investigate some of these issues, we simulate particle trajectories in the inner magnetosphere using large scale magnetic and electric field models. Our goal is to reproduce some of the most salient nose features observed by CODIF, in order to better understand their formation process and from their shape, to deduce more about the large scale electric field and the particle source location and evolution. Our model is a forward particle trajectory tracing. Protons with 90-60 deg pitch angles are traced under the conservation of the first and second adiabatic invariants in time-dependent magnetic and electric fields (Ganushkina et al., 2005).

The particle source distribution have been defined either as a Maxwellian distribution function (situated at $\mathrm{R}=8 R_{E}$, with an MLT location between 05:00 and 19:00 MLT in the equatorial plane) or as a Kappa-like distribution function ( $\mathrm{R}=7 R_{E}, 05: 00-19: 00$ MLT in the equatorial plane). The Kappa-distribution is defined as (Christon et al., 1989):

$f(E)=n\left(\frac{m}{2 \pi k E_{0}}\right)^{3 / 2} \frac{\Gamma(k+1)}{\Gamma(k-1 / 2)}\left(1+\frac{E}{k E_{0}}\right)^{-(k+1)}$

Here $n$ is the particle number density, $m$ is the particle mass, $E_{0}=k_{B} T\left(1-3 / 2^{k)}\right.$ is the particle energy at the peak of the distribution, $k_{B}=1.3807 .10^{-23} \mathrm{~J} / \mathrm{K}$ is the Boltzmann constant, $\mathrm{T}=1 / 3\left(\mathrm{~T}_{/ /}+2 \mathrm{~T}_{\perp}\right), \Gamma$ is the Gamma function:

$\Gamma(k)=(k-1) !$

with $k=5$ in our calculations.

The number density $n$ and perpendicular and parallel temperature estimates were obtained using data from the MPA instrument (Bame et al., 1993) onboard the Los Alamos (LANL) geostationary satellites measuring ions in the energy range $0.1-40 \mathrm{keV}$. The number density and perpendicular and parallel temperatures were then deduced from measurements obtained within $4 \mathrm{~h}$ of local time around midnight. Values were averaged when more than one spacecraft were simultaneously in the same region. When no satellite was in the midnight sector, the data were interpolated linearly. These values were then used as time-dependent boundary conditions.
In the model, we assume an empty magnetosphere before the particle injection, since we wish to study the structure(s) produced by injected particles from the plasma sheet under given electric and magnetic fields. The particles drift velocity is computed as a combination of the velocity due to the $\boldsymbol{E} \times \boldsymbol{B}$ drift and the bounce-averaged velocity due to magnetic drifts. The distribution function at the next time step is obtained assuming conservation of the distribution function along the dynamic trajectory of particles (Liouville theorem), but taking into account the losses caused by charge-exchange. The charge-exchange cross-section is obtained from (Janev and Smith, 1993) and the number density of neutrals from the thermospheric model MSISE 90 (Hedin, 1991). The time of tracing has been set up to $17 \mathrm{~h}$.

The electric field used in our simulations is a $K_{p}$ dependent large-scale Volland-Stern electric field model (Volland, 1973; Stern, 1975).

The Volland Stern (Volland, 1973; Stern, 1975) electric field potential $\Phi_{\text {conv }}$ is given by:

$\Phi_{\text {conv }}=A L^{\gamma} \sin \left(\phi-\phi_{0}\right)$

where $\gamma$ is the shielding factor, $\phi$ the magnetic local time and $\phi_{0}$ is the offset angle from the dawn-dusk meridian. For $A$, which determines the convection electric field intensity, we use a $K_{p}$-dependent function defined by Maynard and Chen (1975):

$A=\frac{0.045}{\left(1-0.159 K_{p}+0.0093 K_{p}^{2}\right)^{3}} \mathrm{kV} / R_{E}^{2}$

where $\gamma=2$ and $\phi_{0}=0$. The $K_{p}$ values used in the model were the observed ones.

Together with this electric field model, a TSY89 (Tsyganenko, 1989) magnetic field model was used.

In our approach, we used the Volland Stern model as defined in the equatorial plane, in which we performed our 2-D particle trajectory simulations. We then projected the calculated fluxes along the Cluster trajectory, off the equatorial plane (for the same L-shell and MLT), assuming flux conservation. To get even more reliable data, we limited the simulated particle fluxes to the corresponding equatorial pitch angle range of particles observed by CODIF at high latitudes. Since the instrument measures particles with pitch angles centered mostly around $90^{\circ}$ all along the pass, we need to calculate the corresponding equatorial pitch angle $\alpha_{\min }$ of the particles presenting a $90^{\circ}$ pitch angle at higher latitudes. In the case of the 22 February 2002 perigee passes we get $\alpha_{\min }=30^{\circ}$.

These simulated fluxes were then directly compared to the fluxes measured by Cluster.

\subsection{Single nose: 22 February 2002 (15:00-17:00 UT)}

As described in Sect. 3.1, a single nose was recorded by CODIF the 22 February 2002, with a characteristic energy at 


\section{February 2002}

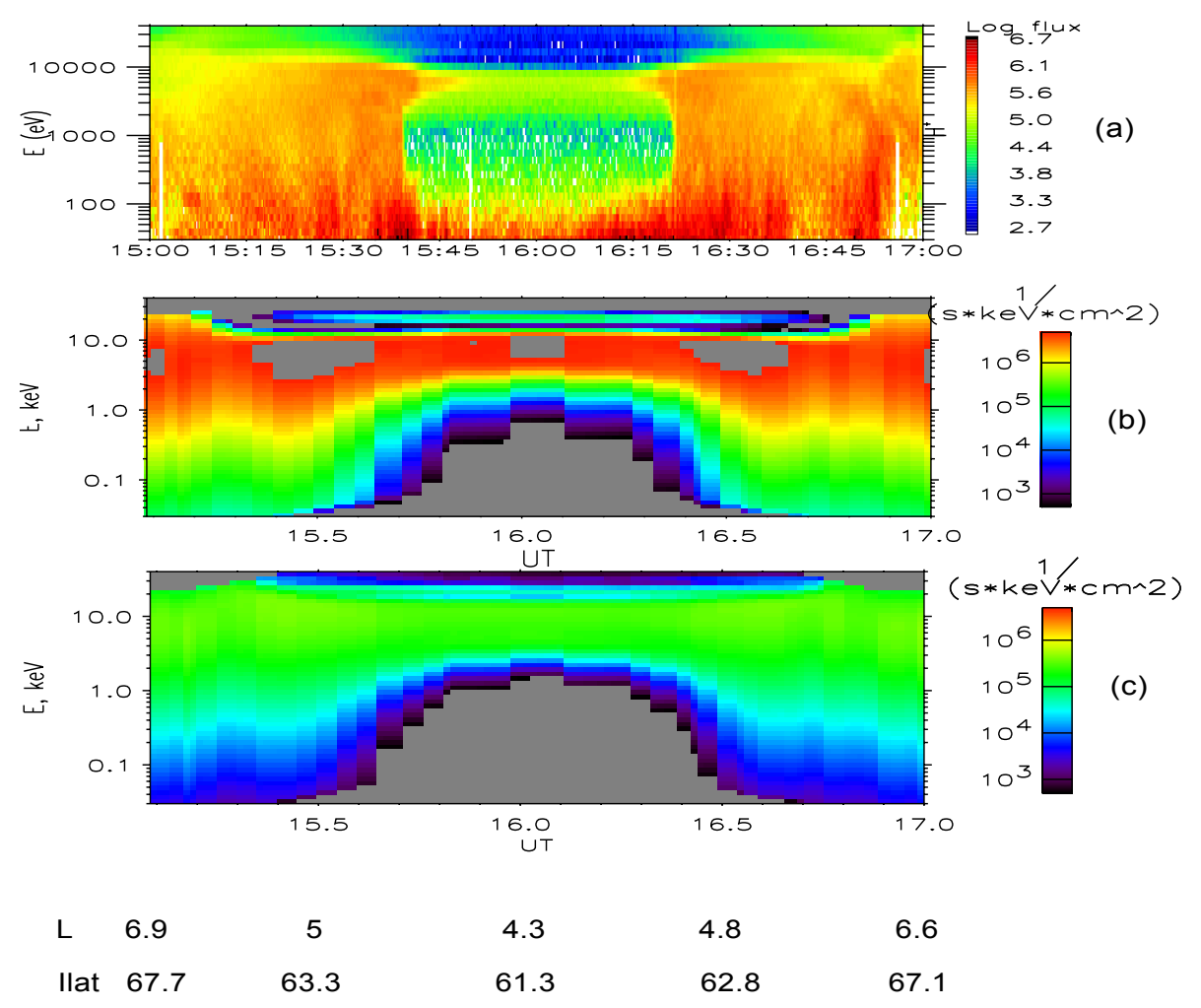

Fig. 10. Real and simulated spectra ( $17 \mathrm{~h}$ of tracing, same flux scale for each panel) for the 22 February 2002 using different parameters models: (a) Observed spectrogram by CODIF/SC4; (b) Simulations under a Volland-Stern electric field and TSY89 magnetic field model, both $K_{p}$ dependents, LANL MPA data as source inputs and assuming a Kappa-like distribution; (c) Same as (b) but assuming a Maxwellian distribution function. The color scale has been defined to be the same in all three panels.

perigee of about $6.5 \mathrm{keV}$. Simulation results for the $22 \mathrm{Febru}-$ ary 2002 event have been presented in Fig. 10.

From top to bottom, the figure presents the $\mathrm{H}^{+}$spectrogram as recorded by CODIF (panel a), the results of the simulation using LANL data as input and a Kappa-like source distribution function under a Volland-Stern electric field and a TSY89 magnetic field, both $K_{p}$ dependent (panel b). Panel (c) shows the same simulation conditions but using a Maxwell-like distribution function. All three panels are plotted using the same flux scale to allow direct comparisons.

Looking at the simulation results, it appears that nose structure can be formed under a global large scale electric field. However, a large part of the particles recorded by CODIF does not appear on the simulated spectra. Moreover, the simulated nose energy range is slightly above the observed one and its energy width is much wider. By comparing panel (b) and (c) of Fig. 10, it seems that, when using a Volland-Stern electric field model, the distribution function of the source plays a crucial role in the features observed at the Cluster location.

The two simulations show very different flux values. Using a Kappa like distribution function in the simulations (panel b) allows a better fit of the CODIF data, quantitatively as well as qualitatively. Panel (c) (using a Maxwellian like distribution function) reveals nose flux values more than one order of magnitude lower than panel (b).

However, even if the LANL/MPA data allows a better estimate of the source parameters, no local time dependence can be deduced concerning the source distribution.

\section{Lack of low energy particles and the sharp flux transition}

At very low energies (up to $\sim 150 \mathrm{eV}$ for protons), Cluster data reveal the presence of a large flux of particles. However, the low energy particles penetration to low L-shells, as well as the sharp flux transition observed by CODIF at 15:39 (inbound) and at 16:21 (outbound) do not appear on the simulated spectrograms.

\subsection{Split nose: 21 November 2001 (21:00-23:00 UT)}

Figure 11 presents the same simulation results than Fig. 10 but for the 21 November 2001 event. The double nose (or "split nose") can be reproduced by using a large scale electric 


\section{November 2001}

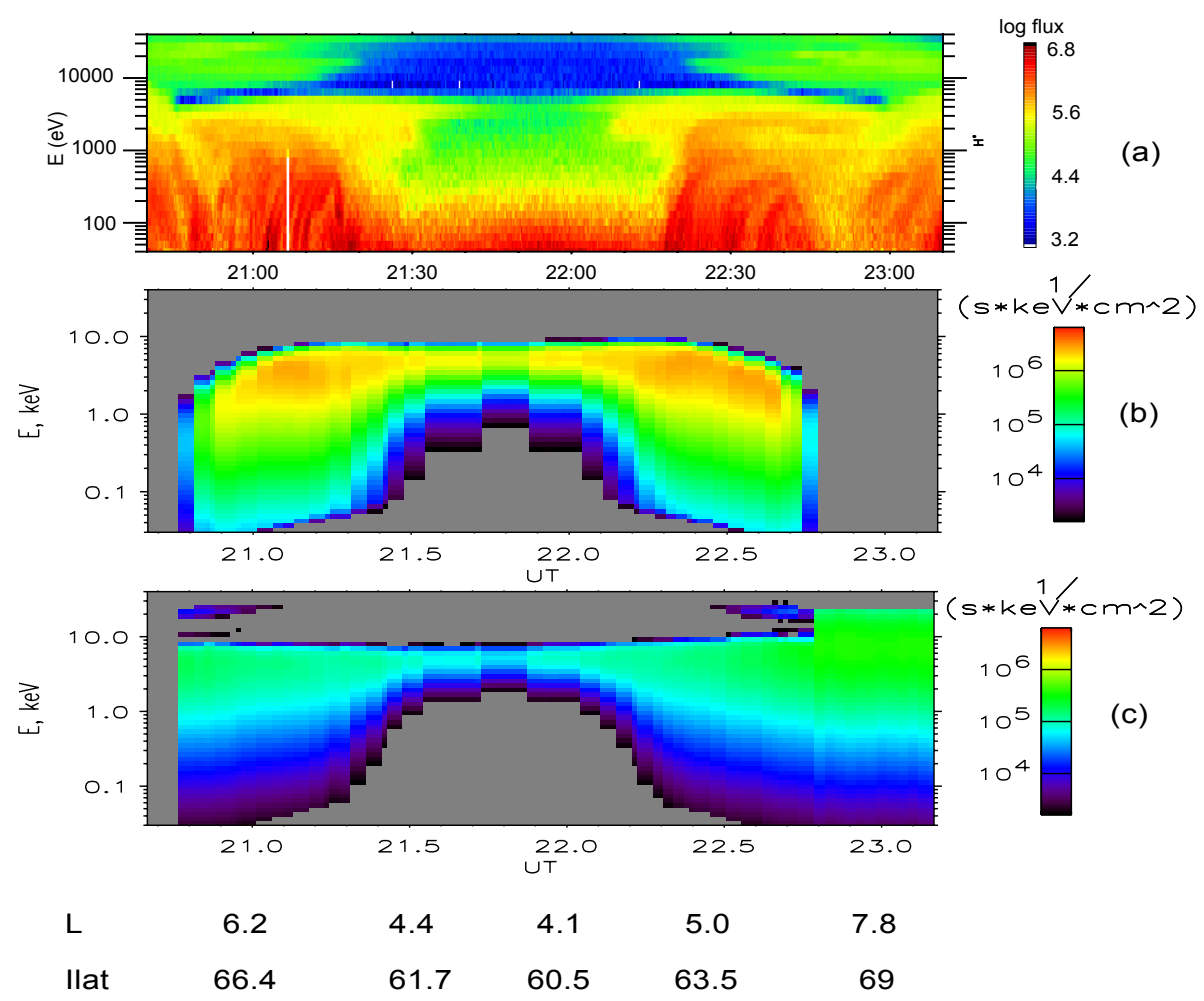

Fig. 11. Same as Fig. 10 but for the 21 November 2001 event.

field model. Panel (b) does not show the high energy nose only because of the flux scale used in the plot. By using a different scale, the high energy peak appears on the spectrogram, even if very low in flux (not shown). The lowest energy nose appears clearly, presenting comparable flux values (panel b). The maxwellian distribution function of the source shows a higher flux for the high energy nose (panel c), even if fluxes are at least 1.5 orders of magnitude lower than the observed ones. The gap is present at $\sim 15 \mathrm{keV}$, i.e. comparable to the one recorded by CODIF.

The low energy part of the spectra (i.e. below $300 \mathrm{eV}$ ) is not reproduced by the simulations. However, our simulations show that the high energy particles observed by CODIF (from $\sim 10$ to $\sim 40 \mathrm{keV}$ ) as well as medium energy particles (below $10 \mathrm{keV}$ ) can be issued from a single injection (and aren't necessarily a trapped population issued from a previous injection).

\subsection{Multi- nose: 11 April 2002 (04:00-07:00 UT)}

Results of the simulations for the 11 April 2002 multi-nose event are presented in Fig. 12.

\section{Influence of the model}

By examining Fig. 12, it appears clearly that none of the simulations is able to reproduce the multi nose structure observed by CODIF. As a consequence, we can conclude that this kind of structure is not a direct consequence of a change in the large scale electric field.

\section{Discussion about the simulations}

\subsection{February 2002 event}

The nose structure reproduced by the model for the 22 February 2002 event is consistent with the theory of the stationary nose structure formation (Shirai et al., 1997). However, comparison with Cluster data reveals that the simulated nose energy range is slightly above the observed one and its energy width is much wider. Moreover, the poleward edge of the nose is much sharper than the simulated one, especially for the lower energy boundary of the structure.

\subsection{Influence of the source parameters}

To understand the influence of the source distribution on the nose energy range and width, we replace the LANL 


\section{April 2002}
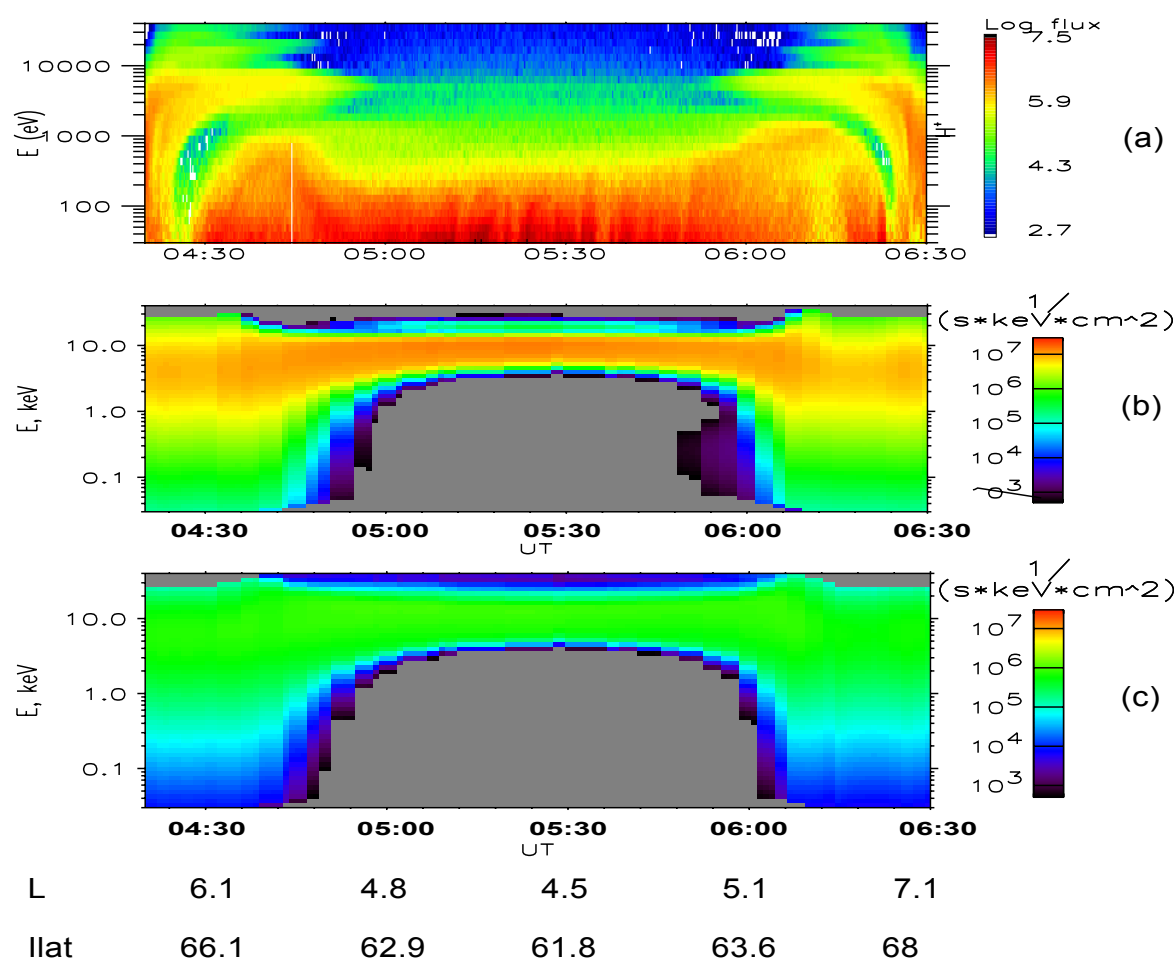

Fig. 12. Same as Fig. 10 but for the 11 April 2002 event.

parameters by using constant source parameters (temperature and density). By studying the influence of a change in one of the source parameters (i.e. density, temperature, distribution function...), we are able to conclude that increasing the density of the source results in a flux increase and increasing the averaged temperature of the source results in a flux decrease at the nose location. As a consequence, changes in the source parameters could affect the nose energy.

\subsection{Role of the plasmasphere}

To better understand the disagreement between CODIF observations and simulations in the lower energy part of the spectrogram, we should consider the role of the plasmasphere. During this event, CODIF onboard SC1 and SC3 are running in RPA mode. The RPA mode ("Retarding Potential Analyzer") allows more accurate measurements in the about 0.7 to $25 \mathrm{eV} / \mathrm{q}$ energy range (Dandouras et al., 2005). As a consequence, CODIF SC1 and SC3 can detect thermal plasma and the exact timing of the plasmapause boundary layer crossing by Cluster. Since during this event the inter-spacecraft separation did not exceed $250 \mathrm{~km}$, we can assume, as a first approach, that the plasmapause boundary layer crossing occurs at almost the same time for the four spacecraft. SC3 shows a clear plasmapause boundary layer crossing at 15:39 UT (Fig. 13), almost simultaneously with the sharp transition seen in the $\mathrm{SC} 4$ proton spectrogram at the plasma sheet energies. We can thus conclude that this abrupt energetic particle flux decrease could be the consequence of Coulomb collision processes (Jordanova et al., 1996), which was not considered in our model. Jordanova et al. (1996) showed that this loss process is important for low energy particles (below $10 \mathrm{keV}$ ) and can lead to particle diffusion, together with a subsequent buildup of the low energy electron population. This process can lead to an "erosion" of the nose structure, narrowing its energy range. Since this process was not considered in our model, the larger than observed nose energy range can, at least partially, be explained. The outbound plasmapause boundary layer crossing does not exactly match with the sharp transition observed on spacecraft 4. However, since this boundary is not as clear as for the inbound (several steps are observed within a short time), it becomes difficult to compare spacecraft 3 RPA data with spacecraft 4 normal mode data. It has to be noted that a nonuniform local time distribution function could also affect the nose energy observed by CODIF and explain partially why simulated nose does not present the same exact energy than the observed one. 
CIS-CODIF SC 3 RPA mode 22/Feb/2002
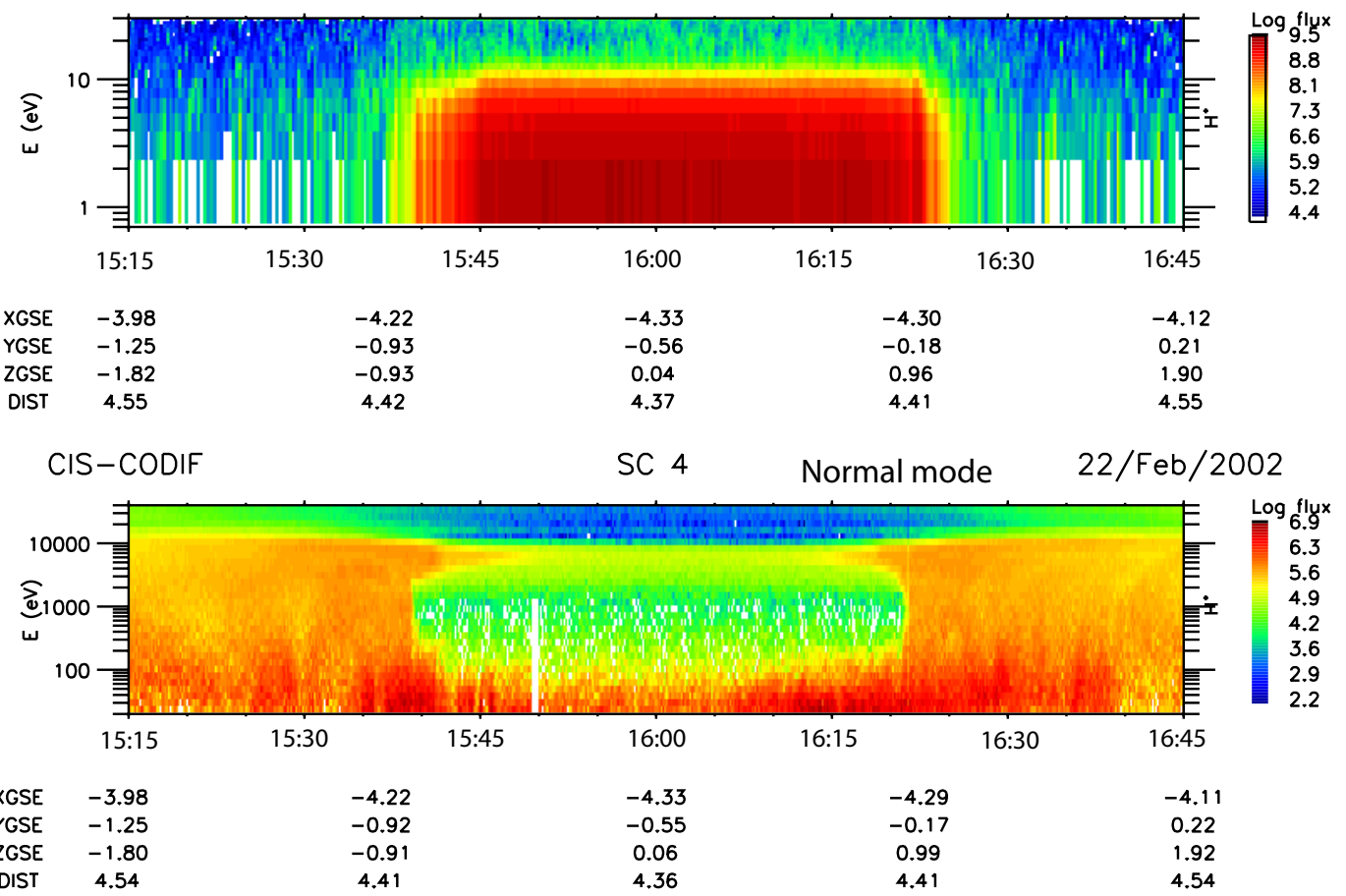

Fig. 13. SC 3 and SC 4 CIS/CODIF proton spectrograms in flux units for the 22 February 2002 event. SC 3 and SC 4 GSE coordinates are indicated below each spectrogram. CODIF SC 3 is running in RPA mode (detection of particles with energy up to $\sim 30 \mathrm{eV}$ ) whereas CODIF SC 4 is running in normal mode (detection of particles with energy from $\sim 25 \mathrm{eV}$ up to $\sim 40 \mathrm{keV}$ ).

7.4 Double nose structure creation during the 21 November 2001 event

Even if the split nose can be roughly reproduced by using a global large scale electric field, some discrepancies with the data still remain: on the one hand, a large part of the low energy population is missing. The reason responsible for such lack of particles is mainly due to the assumption of an empty magnetosphere at the beginning of the simulation. Moreover, our model considers the plasma sheet as the only particle source. However, the ionosphere can be an important source of particles in the inner Magnetosphere (Daglis et al., 1999; Bouhram et al., 2004), and acceleration mechanisms can act on the ionospheric ions to raise the particles from $\sim 1 \mathrm{eV}$ to tens of keV. Since the ionosphere hasn't been considered in the model, it is expected that part of the observed particles with energy below tens of $\mathrm{keV}$ will not be present on the simulation results. The existence of field aligned (resp. anti field aligned) particle populations $\left(\mathrm{H}^{+}\right.$and $\left.\mathrm{O}^{+}\right)$at low energies (below $200 \mathrm{eV}$ ) in the ring current region has been also studied by Vallat et al. (2004). This upwelling population is issued from the ionosphere and observed inside the ring current region. The large amount of field aligned particles in that region points out the importance of the ionosphere as a source of low energy particles at the Cluster perigee location.
7.5 Physical processes responsible for the creation of multi-nose structures: 11 April 2002 case

The simulation conditions are able to reproduce the lowest energy nose (below $10 \mathrm{keV}$ ), even if the simulation results seems to position the nose equatorial edge at lower Lshell than observed, leading to a wider energy range of the nose along the spacecraft orbit track. Based on Explorer 45 data, which revealed that the observed nose location was situated at higher L-shell than expected by calculations, Cowley (1976) argues that the strong pitch angle diffusion processes occurring in that region might limit the inward penetration of particles. Since this loss process hasn't been considered in our model, this could explain why under a Volland Stern electric field model, the calculated nose location appears to be situated automatically at smaller L-shells than the observations.

\section{Low energy population}

A portion of the low energy population recorded by CODIF (below $1 \mathrm{keV}$ ) can be reproduced by using the Volland-Stern electric field model, even if the scale used in Fig. 12 does not allow to see it clearly (except partially in panel b). 


\section{Multi-nose not reproduced by the model}

Multi ion bands have already been recorded in the past. Measurements from CAMMICE/MICS and TIMAS on the Polar satellite have shown multiple discrete energy peaks in the ion energy spectra, over a large range of L-shells (from 3 to 8 ) and energy (a few $\mathrm{keV}$ up to hundreds of $\mathrm{keV}$ ). $\mathrm{Li}$ et al. (2000) have interpreted these features as the result of a time-of-flight effect of the particle's drift around the Earth, i.e. ion drift echoes which can be injected from the plasmasheet by a single propagating time-varying field associated with substorm dipolarization. Li et al. (2000) have been able to reproduce the shape of these bands by introducing in their model an electromagnetic pulse emitted by the substorm associated dipolarization. However, this interpretation is unlikely to explain the bands observed by CODIF on the 11 April 2002, since no dipolarization signature is observed during the $8 \mathrm{~h}$ prior to the event (AE index very low).

Using a realistic electric and magnetic field model (with solar wind data as input), Ebihara et al. (2004) simulated the same event as Li et al. (2000). They were thus able to conclude that these bands were most likely to be produced by two combined process, i.e. a change in the large scale convection electric field (for particles with energy $>3 \mathrm{keV}$ ) and change(s) in the distribution function (e.g. decrease in the number density) in the near Earth magnetotail (for $<3 \mathrm{keV}$ protons). According to Ebihara et al. (2004), a change in the electric field leads to the drift of the stagnation point (i.e. where the sum of the ion drift velocities is zero), and a satellite placed on the closed orbit will detect the gap periodically. This theory is able to explain the substructures observed during the 21 November 2001 event, for which substructures are the result of large scale electric field changes. However, using a realistic large scale electric field model, our simulations couldn't reproduce the bands observed by CODIF during the 11 April 2002 event. As a consequence, the interpretation made by Ebihara et al. (2004) is unlikely to explain CODIF structures in that case. Moreover, using real LANL/MPA data as input in our model, no change in the source distribution function was able to explain the creation of low energy structures.

Sheldon et al. (1998) also recorded multi-nose structures on the CEPPAD/IPS data, and they interpreted these bands as the superposition of a nose structure at $\sim 90 \mathrm{keV}$ and a field aligned beam at $\sim 40 \mathrm{keV}$, which would be issued from the ionosphere and driven to the magnetosphere under the parallel electric field created by the nose ions. However, all bands recorded by CODIF during the 11 April 2002 event present a pitch angle distribution centered on $90^{\circ}$, not consistent with the interpretation of Sheldon et al. (1998) of a field aligned population issued from the ionosphere.

The three bands observed during the outbound part of the orbit could also be the consequence of resonant interactions with local waves, leading to the appearance of gaps at spe- cific energy. However, the Cluster in-situ data do not reveal any wave activity (O. Santolik, private communication).

It has to be noted that, since the electric field depends on the closure of the partial ring current through the ionosphere, a change in the ionospheric conductivity can modify the electric field pattern and thus drive to a twisted drift path of the particle in the ring current region (Fok et al., 2003). Considering that our simulations do not use a self consistent electric field model (i.e. where the electric field created by the closure of the partial ring current through the ionosphere is taken into account), we can conclude that triple nose structures are most probably due to the electric field configuration and to its temporal changes with respect to the ring current closure. Further study checking the validity of this assumption should be published in the future.

\section{Conclusions}

In this paper we present a study of the narrow energy structures observed in the ion spectrograms at medium energies (between 5 and $30 \mathrm{keV}$ ) by Cluster/CODIF at $4 R_{E}$, and their main characteristics were deduced from a statistical study (163 perigee passes of Cluster). Forward particle trajectory modeling has then been performed to understand the main processe(s) involved in the formation of those structures.

We can summarize the main results of this paper within the several points below:

- The detailed analysis of CODIF spectrograms during Cluster perigee pass revealed that several different types of structures are observed at $4 R_{E}$ by CODIF.

- Stationary nose structures are formed by particles issued from the plasmasheet and are the result of particle drift under a global large scale electric field.

- Some gaps can be created within the nose energy range as the result of the antagonistic electric and magnetic field actions, which can drastically reduce the particle drift velocity at this particular energy range. This leads to the creation of double (or rather split) nose features.

- Multi-nose structures (i.e. more than 2) are also observed occasionally by CODIF.

The populations creating these structures are issued from different regions: the ionosphere appears as an important source for low energy particles observed at the Cluster perigee location. The plasmasphere population seems to play a role in scattering particles with energies below $\sim 10 \mathrm{keV}$.

- The statistical study based on more than 160 perigee passes of Cluster (more than one year of data) pointed out the relative distribution of each type of feature as a function of the spacecraft position and the magnetic activity level: 
- Single stationary nose structures (stable between the inbound and the outbound part of the Cluster orbit) are the predominant feature observed at $4 R_{E}$ (within the energy range $\sim 0$ to $40 \mathrm{keV}$ ) during low activity periods, except in the morning sector where "split nose" (as the consequence of a third gap creation) becomes the most frequent feature.

- Double noses are also observed unexpectedly in the evening sector, and the most probable reason(s) for their presence in this MLT sector is a post-midnight location of the source and/or a change in the electric field leading to a twisting drift path of particles.

- The energy range of the first nose (lowest energy one) is decreasing linearly from $\sim 15 \mathrm{keV}$ to $\sim 1 \mathrm{keV}$, counterclockwise, from the pre-noon MLT sector. This is probably due to the appearance of the split nose at MLT 10:00, which creates this artificial jump of the nose energy range in that sector.

- During very active events, the lack of nose structure was the most frequent feature recorded by CODIF.

- Finally, particle trajectory simulations have been performed, aiming to understand the physical processes responsible for the appearance of such features. Results of these simulations pointed out that:

- Single and split noses can be reproduced by a model using a simple large scale electric field.

- However, the extremity of the single nose appears in the simulation results at lower L-shell than observed by Cluster. This can be the consequence of pitch angle diffusion processes occurring in the magnetosphere and which are not taken into account in the model.

- The parameters influencing the energy of the nose are the plasmasheet density and temperature. The MLT distribution of the source is also of prime importance.

- Simulation results reveal weaker fluxes than the ones observed (especially at low energy, i.e. below $\sim 1 \mathrm{keV}$ ), mainly due to the fact than the ionosphere haven't been considered in the model. The ionosphere seems to be a non negligible particle source for low energy ions (22 February 2002).

- The plasma source responsible for the nose formation has an MLT dependence that cannot be totally deduced by the LANL spacecraft data. However, it seems that the MLT location of the source is more likely situated in the post-midnight sector rather than around MLT=00:00.

- Model using a Volland-Stern electric field and a Kappa-like distribution function of the source is the most likely to reproduce the flux values observed by CODIF.
- However, multi-nose structures (i.e. more than two) cannot be reproduced by using a simple large scale electric field. A self consistent electric field model should be considered in the future to better understand the electric field pattern in that region.

Future particle drift trajectory simulations will have to consider additional particle sources (ionosphere) as well as additional loss process (Coulomb interactions) to better reproduce the low energy part of the CODIF spectrograms. The computations will also have to consider a self consistent electric field model to provide a more precise electric field pattern in this region in order to reproduce the multi-nose structures observed by Cluster.

Acknowledgements. The $K_{p}$ index was provided by the World data center for Geomagnetism, Kyoto. The authors are indebted to M. Thomsen who provided us the LANLA/MPA data and they thank O. Santolik for his fruitful comments concerning the Cluster WEC data interpretation.

Topical Editor I. A. Daglis thanks A. Milillo and another referee for their help in evaluating this paper.

\section{References}

Bame, S. J., McComas, D. J., Thomsen, M. F., et al.: Magnetospheric Plasma Analyzer for Spacecraft with Constrained Resources, Rev. Sci. Instr., 64, 1026-1033, 1993.

Bouhram, M., Klecker, B., Miyake, W., Rème, H., Sauvaud, J. -A., Malingre, M., Kistler, L., and Blagau, A.: On the altitude dependence of transversely heated $\mathrm{O}$ distributions in the cusp/cleft, Ann. Geophys., 22, 1787-1798, 2004, http://www.ann-geophys.net/22/1787/2004/.

Buzulukova, N. Yu., Kovrazhkin, R. A., Glazunov, A. L., Sauvaud, J.-A., Ganushkina, N. Yu, and Pulkkinen, T. I.: Stationary Nose Structure of protons in the Inner Magnetosphere: observations by the Ion Instrument onboard the Interball-2 Satellite and Modeling, Cosmic Res., 41(1), 3-12, 2003.

Christon, S. P., Williams, D. J., Mitchell, D. G., Frank, L. A., and Huang, C. Y.: Spectral characteristics of plasma sheet ion and electron populations during undisturbed geomagnetic conditions, J. Geophys. Res., 94(A10), 13 409-13 424, 1989.

Cowley, S. W. H.: Energy transport and diffusion, in: Physics of Solar Planetary environments, edited by: Williams, D. J., p. 585607, AGU, Washington, D. C., 1976.

Daglis, I. A. and Thorne, R. M.: The terrestrial Ring Current: Origin, Formation and Decay, Rev. Geophys., 37, 407, 1999.

Dandouras, I., Pierrard, V. , Goldstein, J., Vallat, C., Parks, G. K., Rème, H., Gouillart, C., Sevestre, F., McCarthy, M., Kistler, L. M., Klecker, B., Korth, A., Bavassano-Cattaneo, M. B., Escoubet, P., and Masson, A.: Multipoint observations of ionic structures in the Plasmasphere by CLUSTER-CIS and comparisons with IMAGE-EUV observations and with Model Simulations, AGU Monograph: Inner Magnetosphere Interactions: New Perspectives from Imaging, 159, 23-53, doi:10.1029/159GM03, 2005.

Ebihara, Y., Yamauchi, M., Nilsson, H., Lundin, R., and Ejiri, M.: Wedge-like dispersion of sub-keV ions in the dayside magnetosphere: Particle simulation and Viking observation, J. Geophys. 
Res., 106(A12), 29571-29584, doi:10.1029/2000JA000227, 2001.

Ebihara, Y., Ejiri, M., Nilsson, H., Sandahl, I., Grande, M., Fennell, J. F., Roeder, J. L., Weimer, D. R., and Fritz, T. A.: Multiple discrete-energy ion features in the inner magnetosphere: 9 February 1998 event, Ann. Geophys., 22, 1297-1304, 2004, http://www.ann-geophys.net/22/1297/2004/.

Ejiri, M., Hoffman, R. A., and Smith, P. H.: Energetic particle penetrations into the inner magnetosphere, J. Geophys. Res., 85, 653663, 1980.

Escoubet, C. P., Fehringer, M., and Goldstein, M.: The Cluster mission, Ann. Geophys., 19, 1197-1200, 2001, http://www.ann-geophys.net/19/1197/2001/.

Fok, M. C., Wolf, R. A., Spiro, R. W., and Moore, T. E.: Comprehensive computational model of the Earth's ring current, J. Geophys. Res., 106(A5), 8417-8424, 2001.

Fok, M. C., Moore, T. E., Wilson, G. R., Perez, J. D., Zhang, X. X., Brandt, P. C., Mitchell, D. G., Roelof, E. C., Jahn, J.-M., Pollock, C. J., and Wolf, R. A.: Global ENA image simulations, Space Sci. Rev., 109, 77-103, 2003.

Ganushkina, N. Yu., Pulkkinen, T. I., Sergeev, V. A., Kubyshkina, M. V., Baker, D. N., Turner, N. E., Grande, M., Kellett, B., Fennell, J., Roeder, J., Sauvaud, J.-A., Fritz, T. A.: Entry of plasma sheet particles into the inner magnetosphere as observed by Polar/CAMMICE, J. Geophys. Res., 105(A11), 25 205-25 220, 2000.

Ganushkina, N. Y., Pulkkinen, T. I., Bashkirov, V. F., Baker, D. N., and Li, X.: Formation of intense nose structures, Geophys. Res. Lett., 28(3), 491-494, 2001.

Ganushkina, N. Yu, Pulkkinen, T. I., and Fritz, T.: Role of substorm-associated impulsive electric fields in the ring current development during storms, Ann. Geophys. Res., 23, 579-591, 2005.

Hedin, A. E.: Extension of the MSIS thermosphere model into middle and lower atmosphere, J. Geophys. Res., 96, 1159-1172, 1991.

Janev, R. K. and Smith, J. J.: Cross sections for collisions processes of hydrogen atoms with electrons, protons, and multiply-charged ions, Atomic and Plasma Materials Interaction Data for Fusion, Int. At. Energ. Agency, 4, 78-79, 1993.

Jordanova, V. K., Kistler, L. M., Kozyra, J. U., Khazanov, G. V., and Nagy, A. F.: Collisional Losses of the Ring Current Ions, J. Geophys. Res., 101, 111-126, 1996.

Jordanova, V. K., Farrugia, C. J., Quinn, J. M., Torbert, R. B., Borovsky, J. E., Sheldon, R. B., and Peterson, W. K.: Simulation of off-equatorial ring current ion spectra measured by Polar for a moderate storm at solar minimum, J. Geophys. Res., 104, 429-436, 1999.

Kovrazhkin, R., Sauvaud, J.-A., and Delcourt, D.: Interball-Auroral observations of the $0.1-12 \mathrm{keV}$ ion gaps in the diffuse auroral zone, Ann. Geophys., 17, 734-742, 1999, http://www.ann-geophys.net/17/734/1999/.

Lennartsson, W., Shelley, E. G., Sharp, R. D., Johnson, R. G., and Balsiger, H.: Some initial ISEE-1 results on the ring current composition and dynamics during the magnetic storm of December 11, 1977, Geophys. Res. Lett., 6(6), 483-486, 1979.

Li, X., Baker, D. N., Temerin, M., Peterson, W. K., and Fennel, J. F.: Multiple discrete energy ion features in the inner magnetosphere: Observations and Simulations, Geophys. Res. Lett.,
27(10), 1447-1450, 2000.

Maggiolo, R., Sauvaud, J.-A., Fontaine, D., Teste, A., Grigorenko, E., Balogh, A., Fazakerley, A., Paschmann, G., and Rème, H.: A multi-satellite study of Accelerated Ionospheric Ion Beams Above The Polar Cap, Ann. Geophys., 24, 1665-1684, 2006, http://www.ann-geophys.net/24/1665/2006/.

Maynard, N. C. and Chen, A. J.: Isolated cold plasma regions: Observations and their relation to possible production mechanisms, J. Geophys. Res., 80, 1009-1013, 1975.

Milillo, A., Orsini, S., and Daglis, I. A.: Empirical model of proton fluxes in the equatorial inner magnetosphere: Development, J. Geophys. Res., 106(A11), 25713-25730, doi:10.1029/2000JA900158, 2001.

Milillo, A., Orsini, S., Delcourt, D. C., Mura, A., Massetti, S., De Angelis, E., and Ebihara, Y.: Empirical Model of proton fluxes in the equatorial inner magnetosphere: 2. Properties and Applications, J. Geophys. Res., 108(A5), 1165, doi:10.1029/2002JA009581, 2003.

Rème, H., Aoustin, C., Bosqued, J. M., Dandouras, I., Lavraud, B., Sauvaud, J. A., Barthe, A., Bouyssou, J., Camus, Th., CoeurJoly, O., Cros, A., Cuvilo, J., Ducay, F., Garbarowitz, Y., Medale, J. L., Penou, E., Perrier, H., Romefort, D., Rouzaud, J., Vallat, C., et al.: First multispacecraft ion measurements in and near the Earth's magnetosphere with the identical Cluster ion spectrometry (CIS) experiment, Ann. Geophys., 1303-1354, 2001.

Sheldon, R. B., Spence, H. E., and Fennel, J. F.: Observation of the $40 \mathrm{keV}$ field aligned ion beams, Geophys. Res. Lett., 25(10), 1617-1620, 1998.

Shirai, H., Maezawa, K., Fujimoto, M., Mukai, T., Saito, Y., and Kaya, N.: Monoenergetic ion drop-off in the inner magnetosphere, J. Geophys. Res., 102, 19873-19882, 1997.

Smith, P. H. and Hoffman, R. A.: Direct observation in the dusk hours of the characteristics of the storm time ring current particles during the beginning of magnetic storms, J. Geophys. Res., 79, 966-971, 1974.

Stern, D. P.: The motion of a proton in the equatorial magnetosphere, J. Geophys. Res., 80, 595-599, 1975.

Tsyganenko, N. A.: A magnetospheric magnetic field model with a warped tail plasma sheet, Planet. Space Sci., 37, 5-20, 1989.

Vallat, C., Dandouras, I., C:son Brandt, P., Mitchell, D. G., Roelof, E. C., deMajistre, R., Rème, H., Sauvaud, J.-A., Kistler, L., Mouikis, C., Dunlop, M., and Balogh, A.: First comparisons of local ion measurements in the inner magnetosphere with ENA magnetospheric image inversions: Cluster-CIS and IMAGE-HENA observations, J. Geophys. Res., 109, A04213, doi:10.1029/2003JA010224, 2004.

Vallat, C., Dandouras, I., Dunlop, M., Balogh, A., Lucek, E., Parks, G., Wilber, M., Roelof, E. C., and Rème, H.: First use of the curlometer technique in the ring current using multi-spacecraft CLUSTER-FGM data: 3-D mapping of the inner magnetosphere currents, Ann. Geophys., 23, 1849-1865, 2005, http://www.ann-geophys.net/23/1849/2005/.

Volland, H.: a semiempirical model of large scale magnetospheric electric field, J. Geophys. Res., 78, 171-180, 1973.

Williams, D. J.: Ring Current and Radiations Belts, Rev. Geophys., 25(3), 570-578, 1987.

Wilken, B., Daly, P. W., Mall, U., Aarsnes, K., Baker, D. N., Belian, R. D., Blake, J. B., Borg, H., Büchner, J., Carter, M., Fennell, J. F., Friedel, R., Fritz, T. A., Gliem, F., Grande, M., Kecskemety, 
K., Kettmann, G., Korth, A., Livi, S., McKenna-Lawlor, S. Mursula, K., Nikutowski, B., Perry, C. H., Pu, Z. Y., Roeder, J., Reeves, G. D., Sarris, E. T., Sandahl, I., Søraas, F., Woch, J., and Zong, Q.-G.: First results from the RAPID imaging energetic particle spectrometer on board Cluster, Ann. Geophys., 19, 1355-1366, 2001,

http://www.ann-geophys.net/19/1355/2001/.
Yamauchi, M., Brandt, P. C., Ebihara, Y., Dandouras, I., Nilsson, H., Lundin, R., Rème, H., Vallat, C., Lindquvist, P.-A., Balogh, A., and Daly, P. W.: Source location of the wedge-like dispersed ring current in the morning sector during a substorm, J. Geophys. Res., 111, A11S09, doi:10.1029/2006JA011621, 2006. 